\title{
Educational fMRI: From the Lab to the Classroom
}

\author{
Mohamed L. Seghier*, Mohamed A. Fahim and Claudine Habak \\ Cognitive Neuroimaging Unit, Emirates College for Advanced Education (ECAE), Abu Dhabi, United Arab Emirates
}

Functional MRI (fMRI) findings hold many potential applications for education, and yet, the translation of fMRI findings to education has not flowed. Here, we address the types of $\mathrm{fMRI}$ that could better support applications of neuroscience to the classroom. This 'educational fMRl' comprises eight main challenges: (1) collecting artifact-free fMRI data in school-aged participants and in vulnerable young populations, (2) investigating heterogenous cohorts with wide variability in learning abilities and disabilities, (3) studying the brain under natural and ecological conditions, given that many practical topics of interest for education can be addressed only in ecological contexts, (4) depicting complex age-dependent associations of brain and behaviour with multi-modal imaging, (5) assessing changes in brain function related to developmental trajectories and instructional intervention with longitudinal designs, (6) providing system-level mechanistic explanations of brain

OPEN ACCESS

Edited by:

Marcel Ruiz-Mejias,

Pompeu Fabra University, Spain

Reviewed by:

Robert Trampel,

Max-Planck-Gesellschaft (MPG),

Germany

Hyemin Han,

University of Alabama, United States

${ }^{*}$ Correspondence:

Mohamed L. Seghier mseghier@gmail.com

Specialty section:

This article was submitted to Educational Psychology, a section of the journal Frontiers in Psychology

Received: 05 September 2019 Accepted: 25 November 2019 Published: 06 December 2019

Citation:

Seghier ML, Fahim MA and Habak C (2019) Educational fMRI: From the Lab to the Classroom.

Front. Psychol. 10:2769. doi: 10.3389/fpsyg.2019.02769 function, so that useful individualized predictions about learning can be generated, (7) reporting negative findings, so that resources are not wasted on developing ineffective interventions, and (8) sharing data and creating large-scale longitudinal data repositories to ensure transparency and reproducibility of $\mathrm{fMRI}$ findings for education. These issues are of paramount importance to the development of optimal fMRI practices for educational applications.

Keywords: education, cognitive neuroscience, functional neuroimaging, translation, application, classroom, variability, reliability

\section{INTRODUCTION}

For more than a century, many teaching methods have been developed and tested, by drawing on the implications of social, cognitive, and developmental psychology for educational practice. Typically, these teaching methods have been implemented in the educational setting without accounting for the workings of the 'black box' of the mind (Byrnes and Vu, 2015). There has been growing interest in how neuroscience can contribute to efficient learning in the classroom (Goswami, 2004; Petitto and Dunbar, 2009; Sousa, 2010; Frith, 2011; Sigman et al., 2014; Thomas et al., 2019a). For example, relevant neuroscience evidence can direct the implementation and design of efficient instruction methods, based on whether the instructions are compatible with how the brain processes information (Immordino-Yang and Gotlieb, 2017; Mayer, 2017; Thomas et al., 2019a). This requires educators to understand the neuroscience literature, and to be able to identify the most useful evidence for the educational context. A large proportion of the neuroscience literature comes from neuroimaging work, in particular findings obtained with functional MRI (fMRI). Even though fMRI is thought of as a single general tool, its 
application varies from one context to another: a better understanding of these applications would be useful for educators to better assess the types of fMRI that are most relevant to questions in education.

The amount of knowledge on how the brain works is growing exponentially, especially for learning-related functions, such as attention, affect, memory, decision-making and control, or for learning-related skills such as reading, writing, number processing, and problem solving (Peelen and Kastner, 2014; Nieder, 2016; Pessoa and McMenamin, 2017; Wandell and Le, 2017; Ng, 2018), and for their interactions with sleep, nutrition and physical activity (Khan and Hillman, 2014; Sigman et al., 2014). It makes sense to capitalize on this rising knowledge to optimize learning practice, especially when deciding on competing teaching models, or when seeking tailored support for individuals with learning difficulties (e.g. Neville et al., 2013). However, neuroscience findings are technical and complex, and they have not been framed within the context of education, so this extensive research has not yet found its impact on education.

Making sense of the wide-ranging fMRI findings, sometimes with inconsistent results across studies, can be challenging, which leads to a wider gap between neuroscience and education (Bowers, 2016). However, appropriate and transparent fMRI methods (Carp, 2012; Soares et al., 2016) that ensure reliable inferences (Bennett and Miller, 2010; Dubois and Adolphs, 2016) can be applied to educationally-relevant contexts. The apparent gap between education and neuroscience is typical of the growth process of any emerging field: evidence from neuroscience has faced resistance in other areas when evaluating its translational potential, with the classic example from clinical neuroscience. Here, we highlight the trends and best practices in $\mathrm{fMRI}$, to maximize its potential implications for education; some of these practices are based on lessons learnt from clinical fMRI (Rosen, 2009).

The term 'clinical fMRI' was coined over two decades ago (Jezzard and Song, 1996; Thulborn et al., 1996) to highlight the potential implications of fMRI findings to the clinical setting (Detre and Floyd, 2001). Other specialized applications such as pharmacological fMRI (Jokeit et al., 2001; Harvey et al., 2018) and foetal- and newborn-fMRI (Hykin et al., 1999; Seghier et al., 2006) have also been developed for clinical applications. The potential use of clinical fMRI was initially met with resistance from clinicians, given the many potential pitfalls and limitations in making robust and reliable fMRI inferences for clinical practice (Desmond and Chen, 2002; Hennig et al., 2003; Haller and Bartsch, 2009; Dubois and Adolphs, 2016; Eklund et al., 2016; Turner, 2016; Roalf and Gur, 2017; Rizzolatti et al., 2018). The most frequent stillpresent critiques of fMRI by clinicians are six-fold: (1) fMRI is too slow, given its low temporal resolution and the inherent hemodynamic delays, (2) it only provides indirect measures (through neurovascular coupling) of neuronal activity, (3) it is preoccupied with the localization of brain "blobs" at arbitrary statistical thresholds with no insight into the biological mechanisms of brain function, (4) it is highly variable, with typically moderate intra-subject consistency and large betweensubject variability, (5) it is notoriously difficult to conduct in vulnerable populations when participant cooperation (e.g. staying still and executing a task) is difficult, and (6) fMRI data is too complex, and it relies on sophisticated processing methods that may complicate result interpretation. Despite this, fMRI has become a useful tool in clinics, as a non-invasive option in addressing certain challenges, such as determining hemispheric dominance in patients, mapping vital brain functions before surgery, visualizing brain reorganization to support recovery, and localizing epileptic foci.

The relevance of clinical fMRI is expected to grow even more so, thanks to recent developments in cutting-edge techniques, and to a new paradigm shift in how fMRI can be applied: from standard static brain maps obtained with subtractive logic on data collected with block designs, to dynamic connectivity maps obtained while participants are doing 'nothing' (at rest) in the scanner. These developments are occurring at both conceptual and methodological levels (e.g. Turner, 2016), and they have opened new horizons in neuroscience, including systems neuroscience, precision neuroscience, and population neuroscience. However, despite these new developments, fMRI is still not seen as a tool of choice by researchers interested in educational topics because it is (1) an expensive technique with high running costs, (2) not portable, (3) not easy to use on young school-aged participants, (4) not flexible enough for large-scale studies or when simultaneous parallel acquisitions in multiple participants are of interest, (5) limited in terms of paradigm designs that can be delivered within a typical scanning environment, and (6) restricted to looking inside the brain while the participant is lying still in a tube, which is not ideal for the typical educational context with active students moving in class. Nevertheless, the unparalleled look inside the brain that fMRI can bring, outweighs these limitations; see for instance recent review about the potential of fMRI in extending our understanding of the neural basis of memory development (Ofen et al., 2019).

To frame fMRI within the educational context, it would make sense to define first the scope of educational neuroscience (or mind, brain and education). Educational neuroscience is a transdisciplinary domain concerned with two complementary questions: how neuroscience evidence can be used to optimize leaning and teaching, and how education can enhance intellectual abilities and change the brain. In a recent systematic thematic analysis of the literature over the last 30 years, three foundational pillars were identified for the field of educational neuroscience that include application, interdisciplinarity, and translation (Feiler and Stabio, 2018). According to some recent models, there are two pathways linking the application and translation of neuroscience to education, one direct pathway that considers the brain as a biological organ that needs to be in the optimal condition to learn, and an indirect pathway mediated by psychology as neuroscience shapes psychological theory and psychology influences education (Thomas et al., 2019a). Current neuroscience evidence is dominated by findings related to the indirect pathway. To increase impact through the direct pathway, neuroscience evidence needs to progress further towards system-level mechanistic explanations of learning, and to adopt a holistic view that contextualizes learning across 
multiple dimensions, encompassing wellbeing, social cognition, affective processing, nutritional factors, genetic factors, sleep, and exercise. This holistic approach involves the blending of diverse disciplines, research methodologies, and paradigms, which integrates multiple levels of analysis to form a multilevel yet coherent explanation for learning, and to inform educational practice and policy (Han et al., 2019). Here, the focus is to frame fMRI within the evolving and transdisciplinary interface of education and neuroscience.

Accordingly, there are many examples of how fMRI research has actually informed educational theories and practices, by providing, for example, biological explanations about brainbehaviour associations during learning and development, e.g. see recent work about the brain correlates of reading acquisition (Chyl et al., 2018; Takashima et al., 2019), and conceptual knowledge in STEM learning (Cetron et al., 2019). A review of this large body of literature can be found elsewhere (e.g. see Sousa, 2010; Butterworth et al., 2011; Sigman et al., 2014; Black et al., 2015; Ozernov-Palchik et al., 2016; ImmordinoYang and Gotlieb, 2017; Thomas et al., 2019a). The current review aims to address a different question: what type of fMRI is most useful for educational purposes? The answer to this question invites this "educational fMRI" to embrace new developments and emerging trends that are taking place in the field of functional neuroimaging and brain mapping. Specifically, this educational fMRI should be concerned with: (1) scanning young school-aged populations, (2) investigating samples with wide variability in learning abilities and disabilities, (3) studying the brain under natural and ecological conditions, (4) multi-modal imaging of brain-behaviour associations, (5) assessing developmental or instruction-induced changes in brain function with longitudinal designs, (6) providing system-level mechanistic explanations of brain function, (7) reporting (unbiased) negative findings, and (8) sharing data and creating large-scale longitudinal databases. A comprehensive list of all available strategies and practices in the current literature is beyond the scope of this review: our goal is to highlight that many ingenious solutions have been proposed in the literature, and that the field is changing rapidly, with many strategies and solutions becoming available to researchers in the near future, despite some of the arduous challenges addressed in this review. Although the abovementioned points may also concern the application of fMRI in other fields, they are discussed here in terms of existing challenges, new opportunities and best practices for fMRI in education.

\section{Scanning Young School-Aged Participants}

Educational fMRI is mainly concerned with studying brain function in young populations, as opposed to the dominant representation of adults in the current fMRI literature. However, carrying-out fMRI in children is generally recognized as more challenging than fMRI in adults. There are many difficulties when scanning children in terms of (1) recruitment, (2) ensuring compliance and participant cooperation, (3) stringent ethical practices to ensure children's safety, (4) age-appropriate designs in task-related fMRI experiments, (5) wide differences in task performance, (6) data distortion in the presence of head motion artifacts, (7) the availability of age-appropriate atlases and customized templates for group analyses, (8) age-dependent changes in brain structure (anatomy) and hemodynamic responses, and (9) interpretation of brain activations that may change with time and expertise. Many reviews in the fields of pediatric and developmental neuroimaging have proposed very useful recommendations on how to effectively scan young participants and process data (e.g. Kotsoni et al., 2006; Church et al., 2010; Greene et al., 2016; Fassbender et al., 2017; Cusack et al., 2018; Wilke et al., 2018). In the paragraphs below, we focus on three major issues pertaining to studying learning and development in children, using fMRI.

The first issue concerns the selection and characterization of participants. As discussed below, young participants generally display higher group heterogeneity compared to typical adult populations, due to differences in age, abilities, expertise, and to familiarity with tasks and research environment. Other issues related to comorbid conditions (Margari et al., 2013; Willcutt et al., 2019), medication status, and clinical assessment need to be taken into account (Greene et al., 2016). In addition, individual differences in hemodynamic responses may vary with participant age (Kozberg and Hillman, 2016), with many studies reporting age-dependent changes in cerebral blood flow (Wu et al., 2016) that could impact on the apparent differences in brain activation between groups of variable age (Vasta et al., 2018). These age-dependent differences in hemodynamic response complicate the interpretation of brain function differences across child groups of differing age (and school year). Age-appropriate flexible response functions or model-free approaches should thus be considered (Cusack et al., 2018). For multi-subject fMRI studies in young learners, high between-subject variability is expected, so it is useful to gather as much information as possible about participants (i.e. demographic and behavioral data), as this can be valuable for optimally modeling data and interpreting results.

The second issue is head motion, which has been widely discussed in previous studies. Children's head motion has the potential to systematically affect individual differences in BOLD changes and in measures of functional connectivity within and across groups (Satterthwaite et al., 2012; Engelhardt et al., 2017; Fassbender et al., 2017). This issue is complicated further, because in-scanner head motion also correlates with many variables of interest such as age, clinical status, cognitive ability, and symptom severity, and hence it has the potential to introduce systematic bias in brain activations and connectivity (Satterthwaite et al., 2019). Some methodological solutions for minimizing head motion have been suggested in this active domain of research, including, for example, the application of prospective motion correction techniques (Zaitsev et al., 2017), robust pre-processing tools (Esteban et al., 2019), the use of screenprinted flexible MRI receive coils for better comfort (Corea et al., 2016; Winkler et al., 2019), or size-optimized coils to increase acceleration in MRI scan acquisition in younger participants (Keil et al., 2011). Furthermore, alternative behavioral procedures used in fMRI for children have been shown to successfully reduce anxiety, improve compliance, and minimize in-scanner head motion in young children, including pre-scan 
training with a mock scanner (de Bie et al., 2010; Li et al., 2019b), watching an introductory video about what to expect before scanning (Szeszak et al., 2016; Waitayawinyu and Wankan, 2016), using head restraints that can be tolerated by children (Fassbender et al., 2017), communicating with participants at regular intervals between runs of the paradigm and checking that they are comfortable (Fassbender et al., 2017), watching low-demand movies during data collection of task-free fMRI data (Vanderwal et al., 2015), and providing real-time visual feedback about head movement (Greene et al., 2018). These procedures should be included in fMRI experiments when scanning school-aged participants.

The third issue is task performance, which is of concern to behavioral testing in general, but it can be more tenuous under fMRI conditions. In addition to making scanning sessions as comfortable as possible for children to maintain attention to the paradigm (Fassbender et al., 2017; Wilke et al., 2018), it is recommended to collect in-scanner performance by administrating active tasks rather than passive tasks to keep participants engaged, and to incorporate strategies that can deal with group performance differences (Church et al., 2010). To maintain engagement, the use of shorter runs is preferable to longer runs. In addition, tasks should be doable by all participants, who may differ in age and abilities; this would ensure that task demand is matched across participants. Realtime monitoring of in-scanner performance helps in ascertaining that children are engaged in the task (Wilke et al., 2018). In addition, positive reinforcement can also help in maintaining a child's motivation to "do well" inside the scanner and improve amenability to the task and performance; these include social rewards such as frequent words of encouragement or tangible rewards. These procedures allow for the collection of highquality artifact-free fMRI data.

\section{Investigating Heterogeneous Cohorts With Wide Variability in (Dis)Abilities}

fMRI provides a remarkable non-invasive tool to study cognition across the lifespan and in vulnerable young populations. These populations are likely to be heterogenous due to various factors that can impact a student's brain during the school years, for example, age- and learning-related changes (Chyl et al., 2018; Caras and Sanes, 2019; Geng et al., 2019). One important question in education is to characterize the brain factors that can explain the wide variability in learning (dis)abilities. Learning capacity and rate vary considerably across students even within the same educational setting, with some students learning quickly while others struggle with learning. For that reason, fMRI in education should pay attention to the individual effect and go beyond the typical aggregate group inferences in fMRI that assume the 'average' brain can fit all sizes. Although this makes it possible to link an average brain to an average behaviour, it ignores any deviation from the mean and treats it as noise. Many fMRI studies have indeed highlighted that this framework is too reductionist, and that between-subject variability is meaningful (e.g. see a recent review by Seghier and Price, 2018). Paying attention to the individual effect is useful when studying brain correlates of skills that are known to vary considerably across students, such as reading skills (for example Seghier et al., 2008; Fischer-Baum et al., 2018; Malins et al., 2018). To fully appreciate variability in brain function, rich demographic and behavioral data are needed to better model and interpret fMRI findings, including accuracy, response times, error types, and learning rates; post-scan debriefing questionnaires can also provide valuable information. These data can be highly useful when mapping functional plasticity in childhood with fMRI (Dennis et al., 2014).

Variability in brain function may result from complex genetic-by-environment interactions, and thus, may contain signatures of individual differences in abilities. For instance, previous fMRI studies have shown that variability in brain activations can be associated with individual differences in many cognitive and behavioral dimensions such as short-term memory capacity, motivational state, learning aptitude, attention shifting efficiency, cognitive flexibility, academic diligence, decision making, inhibitory efficiency during executive functions, and other higher cognitive abilities (Todd and Marois, 2005; Wager et al., 2005; Chuah et al., 2006; Locke and Braver, 2008; Barnes et al., 2014; Armbruster-Genç et al., 2016; Asaridou et al., 2016; Hilger et al., 2017; Fuhrmann et al., 2019). To maximize the usefulness of fMRI for educators, inter-individual variability in brain function should be treated as data rather than noise (Kosslyn et al., 2002; ThompsonSchill et al., 2005; Seghier and Price, 2018). In this context, accurate characterization of the variability in typical populations allows us to derive sound characterisations of the neuronal correlates of learning difficulties at the individual level. In other words, to understand what constitutes atypical processing, we must first understand what can be considered typical, by accurately estimating the typical range of variability in brain function. This issue is crucial to both diagnostic and prognostic purposes (Seghier and Price, 2018).

Furthermore, understating between-subject functional variability recognizes that a given brain function can be sustained by different processing pathways, and the activation of these pathways may vary with individual strategies and preferences (Price and Friston, 2002; Seghier et al., 2008). Individual differences in the activation of these processing pathways may yield less consistently overlapping effects in typical aggregate fMRI group statistics. However, by looking at structure or patterns in the between-subject variability, it becomes possible to decode the different processing pathways that can sustain a given function (Seghier and Price, 2009). One useful framework is to model between-subject variance in activation as a mixture of different subgroups instead of assuming one single homogeneous group: the goal is to maximize similarity within subgroups at the same time as maximizing differences between subgroups. This rationale has been used previously to tease-apart different subgroups of healthy participants who used different strategies to execute the same tasks (Kherif et al., 2009; Cerliani et al., 2017). Previous studies in clinical fMRI have shown the usefulness of understanding variability in brain function to explain interpatient variability in deficit severity and recovery capacity after brain damage (Price et al., 2017), and ultimately, to design tailored individualized interventions and generate accurate 
individualized predictions (Reinkensmeyer et al., 2016). fMRI for education should embrace these emerging trends to assess meaningful individual differences in development, learning, and cognition (Brown, 2017; Foulkes and Blakemore, 2018).

In the same way, when investigating atypical processing in children with learning difficulties such as dyslexia or dyscalculia, it is useful to acknowledge that typical and atypical processing do not always reflect a categorical distinction with clear-cut thresholds (Marquand et al., 2019); but rather, lie on a continuum of the full spectrum of learning and cognitive processing. Atypical processing itself varies (e.g. different types of learning difficulties) (Gomides et al., 2018), and characterization of these sub-types rests upon a better understanding of the variability in brain function, in particular when designing intervention procedures. Accordingly, defining fMRI-based brain markers of learning difficulties at the individual level requires optimal modeling (Thomas et al., 2019b) for parsing the heterogeneity in school-aged young populations and for understanding the comorbidity between learning difficulties (Willcutt et al., 2019). Last but not least, for an individual struggling with learning in one way, neuroscience can advise on the most efficient alternative way, based on the available processing pathways in that individual. This would also be powerful for developing educational applications, because educators are interested in knowing more about the wide variability in learning (dis) abilities, with the ultimate aim of personalizing teaching methods (Immordino-Yang and Gotlieb, 2017).

\section{Studying the Brain Under Natural and Ecological Conditions}

fMRI findings that have the best translational potential are those that can be obtained under experimental conditions that are as close as possible to ecological, or real-world, contexts (Lowe, 2012; Maguire, 2012). Observing the brain while executing time-locked pseudorandomised repeated stimuli might not be the ideal context to fully understand how the brain works in daily-life. In the last decade, some studies have looked at conducting fMRI studies while participants' thoughts wander freely (i.e. resting-state fMRI), are in natural, or in uncontrolled stimulation conditions, such as sleep, mental reasoning, continuous reading, listening to narrative stories, watching movies, and more, (e.g. Bartels and Zeki, 2004; Malinen et al., 2007; Lahnakoski et al., 2012; Wang et al., 2015; see a recent review by Vanderwal et al., 2019). This opportunity to observe the brain in natural contexts is highly appealing to educators looking for biological explanations of mental states that could hinder learning quality, such as, mind wandering, misbehavior, poor memory retention, lack of concentration, demotivation, disinterest, and fatigue, inside the classroom. Naturalistic fMRI experiments can help to ensure that the thoughts or behaviors being investigated are not perturbed or constrained by the imaging protocol, making it possible to engage neural circuits under real-life conditions (Hasson and Honey, 2012; Maguire, 2012). For example, using naturalistic protocols, it was possible to monitor brain activity while participants were playing video games (Mathiak and Weber, 2006) or interacting in natural social scenarios
(Deuse et al., 2016) using for instance hyperscanning methods (scanning more than one person simultaneously) (Wang et al., 2018). Recently, the development of reasoning skills in children aged between 3 and 12 years was mapped with fMRI during movie watching (Richardson et al., 2018). In another example, an fMRI session under natural conditions (watching/listening of natural audio-visual movie tracks) enabled researchers to look at language processing while recording eye gaze trajectories (Hanke et al., 2016). In the context of education, these unconstrained stimulations (e.g. watching video clips) have the potential to engage a wide range of brain systems given the diverse streams of information that are typically contained in movies, which can capture dynamic real-world processing; this would ultimately provide a richer depiction of brain function at the individual level (Jang et al., 2017; Moraczewski et al., 2018). Last but not least, recent studies have also shown the potential of naturalistic approaches in studying vulnerable populations, including individuals with autism spectrum disorder (Rosenblau et al., 2016).

Another widely studied topic in functional neuroimaging of the brain under natural conditions is mind wandering. This has potential applications for educators, since mind wandering has been linked to poor outcomes in a wide range of learning tasks (Smallwood et al., 2007). Moments of mind wandering tend to disrupt memory, comprehension, participation in the classroom, and intellectual functioning (Smallwood and Schooler, 2015), in particular when the external sensory stimuli become uninteresting, repetitive, and familiar. Previous resting-state fMRI studies have shown that mind wandering involves an intricate interplay between different networks, in particular the default-mode network (Raichle et al., 2001). These fMRI findings revealed the different neuronal correlates of mind wandering, which could motivate the development of strategies to minimize mind wandering at inopportune times. This includes the need to update the sensory inflow and make it less predictable in classrooms. Interestingly, some studies have investigated the possibility of controlling and modulating mind wandering using stimulations on core regions of the default mode network (Kajimura et al., 2016).

Although fMRI textbooks still consider standard laboratorybased fMRI paradigms as a better-controlled way of looking at the brain, educational neuroscientists should consider the possibility of studying brain function with fMRI using naturalistic protocols, especially, given the recent sophistication of stimuli and data analysis methods. Some research topics of interest to education can be addressed sensibly only in real-world contexts, which emphasizes the importance of looking at the brain with naturalistic approaches.

\section{Combining fMRI With Other Modalities for Multimodal Brain Mapping}

When studying brain function, we do not have a 'golden technique' that addresses all the questions. There are many methods, including fMRI, each has limitations, but they can often provide complimentary information (Ugurbil, 2018). Given the multifaceted developmental changes that occur in the learner's brain at different levels (microscopic to macroscopic) 
and along multiple dimensions (physiology, structure and function), combining different mapping methods can provide an accurate depiction of such changes and their relationship to cognitive and behavioral growth in the preschool years and beyond (Brown and Jernigan, 2012). Many multimodal protocols have been proposed in the literature, including the widely used combination of fMRI with EEG (Pleisch et al., 2019). Here we focus on MR-based modalities that can be acquired in the same scanning environment while the participant is lying in the scanner. Concurrently acquiring information from fMRI and additional modalities can help to quantify longitudinal changes and between-subject differences in brain function, hemodynamics, and structure (Turner and Geyer, 2014; Reid et al., 2016; Larivière et al., 2019). For example, changes in white matter tract microstructure are not directly seen by fMRI (Giorgio et al., 2010; Slater et al., 2019), so adding a diffusion MRI protocol during the same scanning session would provide an opportunity to assess white matter microstructure and use this information to explain changes in brain function (e.g. Richards et al., 2017).

Indeed, combining fMRI data with anatomy information is tremendously helpful for optimal modeling of brain function (Turner and Geyer, 2014; Higgins et al., 2018). One classic example is the development of (functional) language lateralization in school-aged children (Szaflarski et al., 2006; Groen et al., 2012; Nora et al., 2017), a question better understood if combined with information about the maturation or development of major white mater tracts such as the arcuate fasciculus (Sreedharan et al., 2015; Silva and Citterio, 2017). Recent studies have shown that atypical brain functions combined with information about alterations in brain structure explained better symptom severity in children with ADHD (Zhan et al., 2017; Wu et al., 2019). Another example concerns the evaluation of the brain correlates of math or language learning after instruction or intervention where a combination of anatomy and function information provided more accurate explanations than functional information alone (Supekar et al., 2013; Evans et al., 2015; Thieba et al., 2019), and proven to be useful in explaining the co-occurrence of reading and mathematical difficulties in children (Skeide et al., 2018).

Recent development of non-invasive MR-based protocols has opened many opportunities to provide accurate multiscale and multimodal explanations of brain-behaviour associations, including the assessment of brain perfusion with arterial spin labelling (Leung et al., 2016; Armitage et al., 2017), brain morphology (i.e. brain volume, sulci shape and depth, gray matter density, cortical thickness, myelin and ion density) with multiparametric quantitative MRI (Kim et al., 2017; Carey et al., 2018), and white matter microstructure with diffusion MRI (Lundell et al., 2019). When designing experiments with task-based fMRI paradigms in children, it is recommended if scanning time permits, to add a task-free fMRI run for restingstate network segregation, a diffusion MRI acquisition and a high-resolution anatomical scan. To manage experiment length, some of these acquisition protocols may be completed on a different visit, though acquisition time might no longer be an issue in the future with the emergence of new fast MRI acquisition schemes (LeVan et al., 2018; Polak et al., 2019). Many analysis software packages have made the processing of multimodal MRI data accessible even for non-experts.

\section{Multisession Scanning to Assess Developmental or Post-intervention Changes}

For studying brain-behaviour associations, multisession fMRI acquisitions provide a better framework to address questions that are relevant to education, in particular for questions where age and post-instruction time have a strong impact on brain function (Evans et al., 2016; Brod et al., 2017). Thanks to plasticity, the brain changes dramatically across development and in response to experience: for example, during learning, skill acquisition, or following intervention through behavioral protocols or brain stimulation techniques. Many fMRI studies have demonstrated the possibility to detect changes in brain function and connectivity during the development of reading skills or following intervention in children with reading difficulties (Horowitz-Kraus et al., 2015; Murdaugh et al., 2015; Wise Younger et al., 2017; Smith et al., 2018; Yu et al., 2018; Nugiel et al., 2019), with the opportunity to accurately predict individual behaviour (Scheinost et al., 2019). Typically, intervention-induced time-dependent changes can be characterized in cross-sectional or longitudinal fMRI studies. The latter offer better control of potential confounds or nuisance variables, but sometimes lack statistical power and can be constrained by time and funding. In contrast, cross-sectional studies can help to increase power within a reasonable time window. Researchers and educators should be aware of the limitations of each type of design when assessing intervention-induced changes in brain function (King et al., 2018).

One particular example of multi-session fMRI is the investigation of changes in brain function after intervention with neuromodulation techniques, with the possibility to assess effects at the individual level (Abutalebi et al., 2009; Sebastian et al., 2017). One class of intervention protocols, used mainly in clinical neuroscience, is brain stimulation by transcranial direct current stimulation (tDCS) to modulate cortical excitability and hence to enhance cognition. Many studies have used tDCS to improve cognition in patients with Parkinson's disease, Alzheimer's disease, hemi-neglect, epilepsy, and aphasia (Flöel, 2014; Cappon et al., 2016). The application of electrical stimulations in combination with behavioral intervention can enhance recovery capacity, though studies vary considerably in patient selection, treatment-delivery protocols and outcome-measures (Cappon et al., 2016; Al Harbi et al., 2017). For educational purposes, tDCS has also the potential to facilitate learning, including improving verb learning (Fiori et al., 2018), word reading (Xue et al., 2017), working memory (Berryhill and Jones, 2012), arithmetic problem-solving (Hauser et al., 2016), and in treating children with dyslexia (Costanzo et al., 2019), and autism (Osorio and Brunoni, 2019).

Another intervention protocol comes from neurofeedback procedures (Sitaram et al., 2017) where participant-specific brain-related signal is used as feedback to train the participant in self-regulating brain function (Thibault et al., 2018). This 
protocol can induce brain plasticity by means of self-modulation of brain activity in real time. Specifically, fMRI-based neurofeedback protocols use real-time measures of brain activation as a feedback signal, and this signal can summarize regional brain activations, a multivariate pattern (i.e. decoded neurofeedback), or a connectivity pattern in a targeted network (i.e. connectivity-based neurofeedback); for review see (Watanabe et al., 2017). Neurofeedback protocols have been used for diverse conditions, including ADHD (Zilverstand et al., 2017; Rubia et al., 2019), motor disorders (Liew et al., 2016), and cognitive rehabilitation in stroke populations (Kober et al., 2015; Renton et al., 2017). Another interesting application of fMRI-based neurofeedback is in emotion regulation (Linhartová et al., 2019), where self-regulation of amygdala activity helped participants to improve emotion control and reduce anxiety (Herwig et al., 2019), which is highly beneficial in the educational context given the high burden of many anxiety disorders on children functioning (Schwartz et al., 2019). Before looking at the translational potential of such intervention protocols to education, their effectiveness should be assessed with randomized controlled trials and randomized controlled cross-over trials, as has been conducted for tDCS in stroke survivors (Elsner et al., 2015), tDCS for enhancing working memory capacity in healthy individuals (Ikeda et al., 2019), and neurofeedback in adults with ADHD (Schönenberg et al., 2017).

When using fMRI to assess longitudinal changes to brain function, either during learning or during the course of an intervention, it is important to appreciate the degree of reliability one can get from fMRI studies with children and the different limitations and challenges afforded by longitudinal designs (Vetter et al., 2017; Herting et al., 2018; King et al., 2018; Madhyastha et al., 2018; Telzer et al., 2018). In addition, when assessing statistical significance of longitudinal changes, it is recommended to go beyond reporting values of $p$ (Halsey et al., 2015), because values of $p$ provide poor information about replication (Cumming, 2008). A $p$ indicates only whether a given intervention is working or making a difference, but the effect-size provides an estimate of the size of the change or difference. Estimates of effect sizes and confidence intervals (Cumming, 2014) should be used to provide better estimates of the magnitude and precision of an intervention effect or of a developmental change within or between participants. It is true however that many fMRI studies do not include effect-size estimates, and interventions are frequently selected according to significant effects only, but if the magnitude (effect-size) happens to be small, then this would explain why these interventions have shown little success in clinical or educational settings.

Other alternative methods for generating useful inferences in fMRI rely on Bayesian statistics. Bayesian analyses can be more informative and more flexible than traditional methods when it comes to hypothesis testing, model comparison and parameter estimation (Wagenmakers et al., 2016, 2018). For instance, Bayesian hypothesis testing allows researchers to estimate evidence and monitor its progression as real data are added, with the attractive possibility to take into account prior knowledge and to identify the most useful explanations (i.e. models) given the observed data (Konig and van de Schoot, 2018). Many practical tools have been introduced in the neuroimaging literature to make Bayesian approaches accessible to fMRI users interested in testing the presence of an effect and in model selection (see for example, Rosa et al., 2010; Han and Park, 2018, 2019; Soch and Allefeld, 2018). Bayesian approaches can also help in optimal modeling of imaging biomarkers that may change longitudinally (Aksman et al., 2019), which can open new opportunities to examine effects that vary with age and instruction.

\section{Providing System-Level Mechanistic Explanations of Brain Function}

Many researchers in the field of educational neuroscience have begun to recognize that there is no single brain region or connection that is indicative of individual learning capacity (Frith, 2011). Explanations of learning must therefore be expressed at the system level and be derived from mechanistic accounts, with the ultimate aim to identify the exact brain circuitry that can sustain a given mental process (Kopell et al., 2014; Churchland and Sejnowski, 2017). Brain regions do not operate in isolation: identifying the set of interacting regions (i.e. a brain network) or networks that sustain a given task, provides a biologically-plausible way of explaining brain function and behaviour (Mišić and Sporns, 2016; Mill et al., 2017). This network approach offers a more meaningful explanation of brain function in vulnerable populations: many disorders and learning disabilities are better framed as atypicalities in brain connectivity (Du et al., 2018), including for example, autism (Yahata et al., 2016) and schizophrenia (Friston, 2002). Making inferences at the system level opens new possibilities for understanding and treating brain disorders (Thiel and Zumbansen, 2016), and in understanding brainbehaviour associations. For example, it is possible to derive useful measures or scores with task-based networks to generate individual predictions about concept knowledge in STEM learning (Cetron et al., 2019).

Within the network approach, a recent trend has been to look at brain networks during rest (Lowe, 2012). Resting-state networks are remarkably similar to the networks involved in task execution (Mennes et al., 2010; Tobyne et al., 2018), and examining resting-state networks is very useful, because this at-rest connectivity can (1) shape task-dependent connectivity, (2) reflect, albeit not equivalently, how regions are anatomically connected, and (3) provide markers or signatures related to abilities and skills (Koyama et al., 2011; Laird et al., 2011; Mennes et al., 2011; Sala-Llonch et al., 2012; McFarland, 2017; Dubois et al., 2018; Tobyne et al., 2018; Zhang et al., 2019). This intrinsic connectivity of the brain can predict task performance (Baldassarre et al., 2012), recovery pathways after brain injury (Carter et al., 2012), longitudinal changes (Farah and Horowitz-Kraus, 2019; Zhao et al., 2019), and future learning (Mattar et al., 2018). One alternative suggested in recent work is to combine both task-free and task-related connectivity to derive reliable biomarkers of learning difficulties (Elliott et al., 2019). Recent work has also shown the possibility to derive functional connectome fingerprints (i.e. 'connectotype') to 
discriminate between individuals, to accurately generate individualized predictions (Miranda-Dominguez et al., 2014; Finn et al., 2015; Li et al., 2017), and to better understand the neurobiology of learning disorders (Bailey et al., 2018). With segregated brain networks, there is also the possibility to perform statistics on connections (termed edges), using graph theory analyses (Reijneveld et al., 2007), to quantify some useful connectivity metrics (Rubinov and Sporns, 2010) that can serve to discriminate between participants, tasks, and populations (e.g. Yourganov et al., 2010; Li et al., 2014; Khazaee et al., 2015; Caeyenberghs et al., 2017; Edwards et al., 2018).

Perhaps most interestingly, mapping brain networks can help explain how the brain learns: by looking at network reshaping with age (Song et al., 2014) and learning (Bassett et al., 2011; Fatima et al., 2016; Dresler et al., 2017; Mattar et al., 2018), we are able to understand how network changes and maturation enable learning (Chan et al., 2016; Bogdanov et al., 2017). Armed with these system-level inferences, individual abilities can be predicted, as shown recently in ADHD (Rosenberg et al., 2017), and in predicting memory performance improvement after training (Dresler et al., 2017). Recent applications of this emerging network neuroscience of learning can provide unique insights into adaptive neural processes, the attainment of knowledge, and the acquisition of new skills (Bassett and Mattar, 2017; Bogdanov et al., 2017; Cetron et al., 2019; Zhang et al., 2019). Many studies have highlighted the usefulness of network analyses and the possibility to derive solid biomarkers of brain disorders (Bassett and Bullmore, 2009; Braun et al., 2018; Du et al., 2018); for example for reading difficulties (Bailey et al., 2018; Edwards et al., 2018) and autism (Hong et al., 2019). When generating explanations about both brain structure and function, it is important to keep in mind that the mapping between anatomical networks - anatomical connections, and functional networks - statistical associations between functional responses, is not necessarily a linear one-to-one mapping (Meier et al., 2016; Liang and Wang, 2017). Although both types of connectivity show some similarity, they provide complementary information about the correlates of brain disorders ( $\mathrm{He}$ et al., 2017; McColgan et al., 2017; Vega-Pons et al., 2017), which is an important conceptual issue to keep in mind when analyzing multimodal MRI data in children.

Ideally, inferences at the network or system level should encompass mechanistic models of brain function, or how connections work together, for which models of effective connectivity are needed (Friston, 2009). There is a conceptual distinction between functional connectivity and effective connectivity. Functional connectivity represents the statistical associations between regional timeseries, but there is no information about the direction of the interactions; it establishes that connections, either mono- or poly-synaptic, are present but not their direction of action or causality. This type of connectivity is widely used to derive inferences at the network level as detailed above. In contrast, effective connectivity represents the causal, directed, influences between neurons or neuronal populations and thus, provides estimates for the direction of the effects between regions. Effective connectivity models are key to understanding how brain regions work together and interact to process information (Friston, 2011). Recent approaches at high magnetic fields allow layer-specific activations to be detected, which can ultimately estimate the directions of causation between brain areas (Turner, 2016; Huber et al., 2017). One tool that has been widely used in fMRI in adults is dynamic causal modeling (DCM) (Friston et al., 2003; Seghier et al., 2010; Razi et al., 2017), which allows us to make inferences at the neuronal level through finer modeling of neurovascular coupling (Havlicek et al., 2015) and to compare between different explanations of the same data. The output from DCM can be related to many behavioral outcomes, including classification between typical and atypical participants on an individual (Brodersen et al., 2011) or group basis (Friston et al., 2016; Zeidman et al., 2019), and explaining and predicting behaviour through modeling (Rigoux and Daunizeau, 2015). Providing mechanistic explanations can provide unprecedented understating of how the brain implement a given cognitive process, as shown recently in young children across literacy development (Morken et al., 2017).

Armed with these biologically-based mechanistic accounts, it is possible to understand how typical processing is implemented in the brain and how atypical behaviour can emerge, with the potential to define efficient intervention protocols. Mechanistic explanations of brain function are needed for future development of individualized tools and interventions in education. To illustrate this rationale, we can consider the example of an acquired skill like word reading: if we have a mechanistic model that includes the brain areas that sustain reading skills, how these areas communicate together, how behavioral manipulations (e.g. word frequency, familiarity, imageability, sensory modality) modulate the interactions between different subsystems (language, executive, attentional, memory and control), how the reading system changes with expertise, and the size of typical between-subject variability in normal function, then we should be able to make predictions about how normal reading should proceed, the optimal conditions to activate the reading system, and the alternative reading pathways and the potential interventions that can be administrated to learners who struggle with reading.

\section{Reporting Negative fMRI Findings}

The selective publication of positive effects is a well-known bias in the neuroimaging literature that is damaging not only to the integrity of science but also to its ingenuity in solving problems (Ioannidis, 2005). This problem may lead to the perpetuation of (false) positive effects until they become erroneously accepted as fact (Nissen et al., 2016), which leads to misrepresentation or misunderstanding by the media or the general public (Gonon et al., 2011), in particular, when it involves topics of great interest to the general public, such as education and when providing a neuroscience explanation of such effects (Weisberg et al., 2008). Thus, a shift is needed to encourage the publication of null or negative results. Any translational effort to the classroom must consider the balance between what can or cannot be done and what works or does not work in brain research. Negative findings are becoming the missing piece in the neuroscience literature (Pfeffer and Olsen, 2002; 
Schooler, 2011; Parsey, 2018) but they are needed to derive the most unbiased of scientific practices that can help to bridge the gap between neuroscience and education. Without publication of sound and relevant negative findings, the educational neuroscience literature will be skewed, and the correction of false positives difficult; this may lead to time and resources wasted on developing ineffective teaching methods that happen to be based on skewed or false brain research findings.

It is in this context that educational fMRI should emphasize the importance of replication studies. This would help to test findings in different environments (site, scanner, sequence) and samples. Replication studies can help to explain findings that are reliable and robust, along with findings that can be explained by other confounds. Another practice that can help minimize this bias in publication of positive findings and improve transparency is preregistration (Gorgolewski and Poldrack, 2016). This entails plans and predictions being registered prior to data collection, which helps to avoid the practice of selective reporting of desirable findings based on exploratory analyses. Having a proper research data management plan will also help to improve rigor and reproducibility (Borghi and Van Gulick, 2018) when using fMRI on children. Last but not least, data sharing and data repositories can also provide another solution to this issue by making data available for validating previous reports, testing new hypotheses, or aggregating with other datasets to increase statistical power.

There are many reasons why some positive findings have not always been replicated, including differences in experimental protocols (Carp, 2012), different processing tools (Bowring et al., 2019), different statistical manipulations (Nieuwenhuis et al., 2011; Woo et al., 2014; Eklund et al., 2016), limited statistical power with small sample sizes (Button et al., 2013; Lorca-Puls et al., 2018), and high variability in fMRI responses (Bennett and Miller, 2010; Dubois and Adolphs, 2016). Another overlooked issue concerns how researchers interpret their findings, especially when it comes to attributing a given function or role to an activated brain region, using 'reverse' inference (Poldrack, 2006). Inconsistencies are not always about the localization of effects, but in the interpretation of their function, which is usually carried out by manipulating different comparisons between conditions (contrasts, masking, conjunctions...etc.). Before considering positive or negative results across studies, it is necessary to appraise how researchers have assigned functions or cognitive processes to specific brain regions. In sum, educators should not overlook replication studies and the many fMRI studies with negative results.

\section{Data Sharing and fMRI Data Repositories}

The typical sample size in multi-subject task-based fMRI studies is around 16-30 participants, usually predefined arbitrarily or based on power analyses. This allows for inferences at the group level, but it might not be enough for optimal replicability (Turner et al., 2018) when dealing with small population effect sizes, heterogenous groups, individual analyses, or in testing the influence of numerous demographic and behavioral variables on brain function. In that context, data repositories and databases offer an exciting opportunity for data sharing and mining, and for testing specific hypothesis with hundreds of brain scans. This would help to ensure high statistical power (Button et al., 2013) and to generate robust neuroscience findings while taking into account the impact of many confounds (Nichols et al., 2017). There has been a call in the neuroimaging community to support data sharing, and in response, databases have been established with the ultimate aim of depicting comprehensive models of typical and atypical brain function. The gains are not only in improving reproducibility and reliability (Zuo et al., 2014), but also in devising useful models that can predict behaviour in heterogeneous populations (Mueller et al., 2005; Ofori et al., 2016; Satterthwaite et al., 2016; Thompson et al., 2017). Access to big data provides the opportunity to conquer the problem of a lack of normative data in assessing the range of brain function and anatomy, and this would open new avenues for neuroimaging research for educational applications.

Shared data can also be utilized in meta-analyses to inform future fMRI studies and motivate the development of new intervention protocols. Previous meta-analysis studies provided very useful insights about many cognitive processes (see Houde et al., 2010; Pollack et al., 2015; Han, 2017; Yaple and Arsalidou, 2018; Bottenhorn et al., 2019), with the possibility to generate summary maps with high statistical validity using for instance activation likelihood estimation (ALE). This ALE meta-analysis takes into account the spatial uncertainty due to the intersubject and between-template variability of fMRI foci reported from different experiments. Meta-analysis in neuroimaging can take the form of image-based or coordinate-based analysis (Muller et al., 2018). For image-based meta-analysis, users can take advantage of the online OpenNeuro database that contains real task-based fMRI data (Poldrack et al., 2013) and the Neurovault database that contains unthresholded whole-brain statistical images (Gorgolewski et al., 2015). Another useful open tool is NeuroSynth (Yarkoni et al., 2011), a platform for large-scale automated synthesis of fMRI data. Armed with this tool, educators for instance can explore summary maps over thousands of studies about a concept or function of interest, which can help in generating prior knowledge or testable hypotheses for further investigations.

These neuroimaging databases have been supported by the neuroimaging community for diverse clinical applications, including research in Alzheimer disease (Mueller et al., 2005), schizophrenia (Wang et al., 2016), and Parkinson's disease (Ofori et al., 2016). On the other hand, analogous databases on the many known learning disabilities with applications for learning and education are scarce, though some interesting initiatives exist for autism spectrum disorder (Di Martino et al., 2014; Payakachat et al., 2016), ADHD (Hoogman et al., 2019), and dyslexia (Lyytinen et al., 2015). It is also interesting for researchers in educational neuroscience to be involved in initiatives that investigate educationally-relevant neurodevelopmental questions (Gao et al., 2019; Li et al., 2019a) in order to understand healthy brain development (Satterthwaite et al., 2016), including The Baby Connectome Project (Howell et al., 2019), and the Lifespan Human Connectome Project in Aging (Bookheimer et al., 2019). Sharing data should be encouraged in educational 
fMRI to respond to the increasingly recognized need for transparent and reproducible neuroscience (Eickhoff et al., 2016).

\section{CONCLUSION}

Functional neuroimaging protocols are evolving continuously in their sophistication and flexibility to expand the range of research questions and inference levels. Best practice in this ever-expanding field is improving, which will ultimately help to ensure transparent and reliable neuroscience evidence and to maximize the translational potential of neuroimaging findings to education. Although some of the issues discussed here are not specific to educational fMRI, any progress in them will directly impact on the translational potential of fMRI findings to education. Educators, whether interested in conducting neuroscience experiments or searching for the best and most useful neuroscience evidence, need to pay attention to those developments. This is critical when educators are looking for novel alternative intervention methods for students struggling to learn, as the field moves from standardized intervention protocols to targeted individualized intervention strategies, comprising both behavioral therapies and non-invasive brain stimulation. Educators and neuroscientists interested in educational questions should move beyond simplistic correlational

\section{REFERENCES}

Abutalebi, J., Rosa, P. A., Tettamanti, M., Green, D. W., and Cappa, S. F. (2009). Bilingual aphasia and language control: a follow-up fMRI and intrinsic connectivity study. Brain Lang. 109, 141-156. doi: 10.1016/j.bandl.2009.03.003

Aksman, L. M., Scelsi, M. A., Marquand, A. F., Alexander, D. C., Ourselin, S., Altmann, A., et al. (2019). Modeling longitudinal imaging biomarkers with parametric Bayesian multi-task learning. Hum. Brain Mapp. 40, 3982-4000. doi: $10.1002 / \mathrm{hbm} .24682$

Al Harbi, M. F., Armijo-Olivo, S., and Kim, E. S. (2017). Transcranial direct current stimulation (tDCS) to improve naming ability in post-stroke aphasia: a critical review. Behav. Brain Res. 332, 7-15. doi: 10.1016/j.bbr.2017.05.050

Armbruster-Genç, D. J., Ueltzhöffer, K., and Fiebach, C. J. (2016). Brain signal variability differentially affects cognitive flexibility and cognitive stability. J. Neurosci. 36, 3978-3987. doi: 10.1523/JNEUROSCI.2517-14.2016

Armitage, P. A., Skipper, N., Connolly, D. J., and Griffiths, P. D. (2017). A qualitative comparison of arterial spin labelling and dynamic susceptibility contrast MRI in 52 children with a range of neurological conditions. Br. J. Radiol. 90:20160495. doi: 10.1259/bjr.20160495

Asaridou, S. S., Takashima, A., Dediu, D., Hagoort, P., and McQueen, J. M. (2016). Repetition suppression in the left inferior frontal gyrus predicts tone learning performance. Cereb. Cortex 26, 2728-2742. doi: 10.1093/cercor/ bhv126

Bailey, S. K., Aboud, K. S., Nguyen, T. Q., and Cutting, L. E. (2018). Applying a network framework to the neurobiology of reading and dyslexia. J. Neurodev. Disord. 10:37. doi: 10.1186/s11689-018-9251-z

Baldassarre, A., Lewis, C. M., Committeri, G., Snyder, A. Z., Romani, G. L., and Corbetta, M. (2012). Individual variability in functional connectivity predicts performance of a perceptual task. Proc. Natl. Acad. Sci. USA 109, 3516-3521. doi: 10.1073/pnas.1113148109

Barnes, K. A., Anderson, K. M., Plitt, M., and Martin, A. (2014). Individual differences in intrinsic brain connectivity predict decision strategy. J. Neurophysiol. 112, 1838-1848. doi: 10.1152/jn.00909.2013

Bartels, A., and Zeki, S. (2004). Functional brain mapping during free viewing of natural scenes. Hum. Brain Mapp. 21, 75-85. doi: 10.1002/hbm.10153 approaches, and embrace these new trends of multimodal longitudinal designs, along with the use of advanced methods that can estimate causality in brain change to derive systemlevel mechanistic explanations of brain-behaviour associations. This will ultimately help to better understand individual differences, heterogeneity in learner profiles, and the co-occurrence of deficits and comorbidities. The issues highlighted in this succinct review are paramount to the development of optimal fMRI practices for school-aged young individuals, and to ensure that what gets pedagogically evaluated, is top-quality fMRI evidence.

\section{AUTHOR CONTRIBUTIONS}

MS helped in conceptualization, writing, and in original draft preparation. MS, MF, and $\mathrm{CH}$ helped in editing, revising, and in funding acquisition. All authors reviewed and approved the final version of the manuscript.

\section{FUNDING}

This work was funded by the ECAE's Research Office (grant numbers: 30-2017, GP-08-2019, and GP-04-2019).

Bassett, D. S., and Bullmore, E. T. (2009). Human brain networks in health and disease. Curr. Opin. Neurol. 22, 340-347. doi: 10.1097/WCO. 0b013e32832d93dd

Bassett, D. S., and Mattar, M. G. (2017). A network neuroscience of human learning: potential to inform quantitative theories of brain and behavior. Trends Cogn. Sci. 21, 250-264. doi: 10.1016/j.tics.2017.01.010

Bassett, D. S., Wymbs, N. F., Porter, M. A., Mucha, P. J., Carlson, J. M., and Grafton, S. T. (2011). Dynamic reconfiguration of human brain networks during learning. Proc. Natl. Acad. Sci. USA 108, 7641-7646. doi: 10.1073/ pnas. 1018985108

Bennett, C. M., and Miller, M. B. (2010). How reliable are the results from functional magnetic resonance imaging? Ann. N. Y. Acad. Sci. 1191, 133-155. doi: $10.1111 / j .1749-6632.2010 .05446 . x$

Berryhill, M. E., and Jones, K. T. (2012). tDCS selectively improves working memory in older adults with more education. Neurosci. Lett. 521, 148-151. doi: 10.1016/j.neulet.2012.05.074

Black, J. M., Myers, C. A., and Hoeft, F. (2015). The utility of neuroimaging studies for informing educational practice and policy in reading disorders. New Dir. Child Adolesc. Dev. 2015, 49-56. doi: 10.1002/cad.20086

Bogdanov, P., Dereli, N., Dang, X. H., Bassett, D. S., Wymbs, N. F., Grafton, S. T., et al. (2017). Learning about learning: mining human brain sub-network biomarkers from fMRI data. PLoS One 12:e0184344. doi: 10.1371/ journal.pone.0184344

Bookheimer, S. Y., Salat, D. H., Terpstra, M., Ances, B. M., Barch, D. M., Buckner, R. L., et al. (2019). The lifespan human connectome project in aging: an overview. NeuroImage 185, 335-348. doi: 10.1016/j.neuroimage.2018.10.009

Borghi, J. A., and Van Gulick, A. E. (2018). Data management and sharing in neuroimaging: practices and perceptions of MRI researchers. PLoS One 13:e0200562. doi: 10.1371/journal.pone.0200562

Bottenhorn, K. L., Flannery, J. S., Boeving, E. R., and Riedel, M. C. (2019) Cooperating yet distinct brain networks engaged during naturalistic paradigms: a meta-analysis of functional MRI results. Network Neurosci. 3, 27-48. doi: 10.1162/netn_a_00050

Bowers, J. S. (2016). The practical and principled problems with educational neuroscience. Psychol. Rev. 123, 600-612. doi: 10.1037/rev0000025 
Bowring, A., Maumet, C., and Nichols, T. E. (2019). Exploring the impact of analysis software on task fMRI results. Hum. Brain Mapp. 40, 3362-3384. doi: $10.1002 / \mathrm{hbm} .24603$

Braun, U., Schaefer, A., Betzel, R. F., Tost, H., Meyer-Lindenberg, A., and Bassett, D. S. (2018). From maps to multi-dimensional network mechanisms of mental disorders. Neuron 97, 14-31. doi: 10.1016/j.neuron.2017.11.007

Brod, G., Bunge, S. A., and Shing, Y. L. (2017). Does one year of schooling improve children's cognitive control and alter associated brain activation? Psychol. Sci. 28, 967-978. doi: 10.1177/0956797617699838

Brodersen, K. H., Schofield, T. M., Leff, A. P., Ong, C. S., Lomakina, E. I., Buhmann, J. M., et al. (2011). Generative embedding for model-based classification of fMRI data. PLoS Comput. Biol. 7:e1002079. doi: 10.1371/ journal.pcbi.1002079

Brown, T. T. (2017). Individual differences in human brain development. Wiley Interdiscip. Rev. Cogn. Sci. 8:e1389. doi: 10.1002/wcs.1389

Brown, T. T., and Jernigan, T. L. (2012). Brain development during the preschool years. Neuropsychol. Rev. 22, 313-333. doi: 10.1007/s11065-012-9214-1

Butterworth, B., Varma, S., and Laurillard, D. (2011). Dyscalculia: from brain to education. Science 332, 1049-1053. doi: 10.1126/science.1201536

Button, K. S., Ioannidis, J. P., Mokrysz, C., Nosek, B. A., Flint, J., Robinson, E. S., et al. (2013). Power failure: why small sample size undermines the reliability of neuroscience. Nat. Rev. Neurosci. 14, 365-376. doi: 10.1038/ nrn3475

Byrnes, J. P., and Vu, L. T. (2015). Educational neuroscience: definitional, methodological, and interpretive issues. Wiley Interdiscip. Rev. Cogn. Sci. 6, 221-234. doi: 10.1002/wcs.1345

Caeyenberghs, K., Verhelst, H., Clemente, A., and Wilson, P. H. (2017). Mapping the functional connectome in traumatic brain injury: what can graph metrics tell us? NeuroImage 160, 113-123. doi: 10.1016/j.neuroimage.2016.12.003

Cappon, D., Jahanshahi, M., and Bisiacchi, P. (2016). Value and efficacy of transcranial direct current stimulation in the cognitive rehabilitation: a critical review since 2000. Front. Neurosci. 10:157. doi: 10.3389/fnins.2016.00157

Caras, M. L., and Sanes, D. H. (2019). Neural variability limits adolescent skill learning. J. Neurosci. 39, 2889-2902. doi: 10.1523/JNEUROSCI.2878-18.2019

Carey, D., Caprini, F., Allen, M., Lutti, A., Weiskopf, N., Rees, G., et al. (2018). Quantitative MRI provides markers of intra-, inter-regional, and age-related differences in young adult cortical microstructure. NeuroImage 182, 429-440. doi: 10.1016/j.neuroimage.2017.11.066

Carp, J. (2012). The secret lives of experiments: methods reporting in the fMRI literature. NeuroImage 63, 289-300. doi: 10.1016/j.neuroimage. 2012.07.004

Carter, A. R., Shulman, G. L., and Corbetta, M. (2012). Why use a connectivitybased approach to study stroke and recovery of function? NeuroImage 62, 2271-2280. doi: 10.1016/j.neuroimage.2012.02.070

Cerliani, L., Thomas, R. M., Aquino, D., Contarino, V., and Bizzi, A. (2017). Disentangling subgroups of participants recruiting shared as well as different brain regions for the execution of the verb generation task: a data-driven fMRI study. Cortex 86, 247-259. doi: 10.1016/j.cortex.2016.11.017

Cetron, J. S., Connolly, A. C., Diamond, S. G., May, V. V., Haxby, J. V., and Kraemer, D. J. M. (2019). Decoding individual differences in STEM learning from functional MRI data. Nat. Commun. 10:2027. doi: 10.1038/ s41467-019-10053-y

Chan, J. S., Wang, Y., Yan, J. H., and Chen, H. (2016). Developmental implications of children's brain networks and learning. Rev. Neurosci. 27, 713-727. doi: 10.1515/revneuro-2016-0007

Chuah, Y. M., Venkatraman, V., Dinges, D. F., and Chee, M. W. (2006). The neural basis of interindividual variability in inhibitory efficiency after sleep deprivation. J. Neurosci. 26, 7156-7162. doi: 10.1523/JNEUROSCI. 0906-06.2006

Church, J. A., Petersen, S. E., and Schlaggar, B. L. (2010). The "task B problem" and other considerations in developmental functional neuroimaging. Hum. Brain Mapp. 31, 852-862. doi: 10.1002/hbm.21036

Churchland, P. S., and Sejnowski, T. J. (2017). The computational brain. Cambridge, MA: MIT Press.

Chyl, K., Kossowski, B., Dębska, A., Łuniewska, M., Banaszkiewicz, A., Żelechowska, A., et al. (2018). Prereader to beginning reader: changes induced by reading acquisition in print and speech brain networks. J. Child Psychol. Psychiatry 59, 76-87. doi: 10.1111/jcpp.12774
Corea, J. R., Flynn, A. M., Lechêne, B., Scott, G., Reed, G. D., Shin, P. J., et al. (2016). Screen-printed flexible MRI receive coils. Nat. Commun. 7:10839. doi: $10.1038 /$ ncomms10839

Costanzo, F., Rossi, S., Varuzza, C., Varvara, P., Vicari, S., and Menghini, D. (2019). Long-lasting improvement following tDCS treatment combined with a training for reading in children and adolescents with dyslexia. Neuropsychologia 130, 38-43. doi: 10.1016/j.neuropsychologia.2018.03.016

Cumming, G. (2008). Replication and p intervals: p values predict the future only vaguely, but confidence intervals do much better. Perspect. Psychol. Sci. 3, 286-300. doi: 10.1111/j.1745-6924.2008.00079.x

Cumming, G. (2014). The new statistics: why and how. Psychol. Sci. 25, 7-29. doi: $10.1177 / 0956797613504966$

Cusack, R., McCuaig, O., and Linke, A. C. (2018). Methodological challenges in the comparison of infant fMRI across age groups. Dev. Cogn. Neurosci. 33, 194-205. doi: 10.1016/j.dcn.2017.11.003

de Bie, H. M., Boersma, M., Wattjes, M. P., Adriaanse, S., Vermeulen, R. J., Oostrom, K. J., et al. (2010). Preparing children with a mock scanner training protocol results in high quality structural and functional MRI scans. Eur. J. Pediatr. 169, 1079-1085. doi: 10.1007/s00431-010-1181-z

Dennis, M., Spiegler, B. J., Simic, N., Sinopoli, K. J., Wilkinson, A., Yeates, K. O., et al. (2014). Functional plasticity in childhood brain disorders: when, what, how, and whom to assess. Neuropsychol. Rev. 24, 389-408. doi: 10.1007/s11065-014-9261-x

Desmond, J. E., and Chen, A. S. H. (2002). Ethical issues in the clinical application of fMRI: factors affecting the valaidity and interpretation of activations. Brain Cogn. 50, 482-497. doi: 10.1016/S0278-2626(02)00531-6

Detre, J. A., and Floyd, T. F. (2001). Functional MRI and its applications to the clinical neurosciences. Neuroscientist 7, 64-79. doi: 10.1177/ 107385840100700110

Deuse, L., Rademacher, L. M., Winkler, L., Schultz, R. T., Gründer, G., and Lammertz, S. E. (2016). Neural correlates of naturalistic social cognition: brain-behavior relationships in healthy adults. Soc. Cogn. Affect. Neurosci. 11, 1741-1751. doi: 10.1093/scan/nsw094

Di Martino, A., Yan, C. G., Li, Q., Denio, E., Castellanos, F. X., Alaerts, K., et al. (2014). The autism brain imaging data exchange: towards a large-scale evaluation of the intrinsic brain architecture in autism. Mol. Psychiatry 19, 659-667. doi: 10.1038/mp.2013.78

Dresler, M., Shirer, W. R., Konrad, B. N., Müller, N. C. J., Wagner, I. C., Fernández, G., et al. (2017). Mnemonic training reshapes brain networks to support superior memory. Neuron 93, 1227-1235. doi: 10.1016/j. neuron.2017.02.003

Du, Y., Fu, Z., and Calhoun, V. D. (2018). Classification and prediction of brain disorders using functional connectivity: promising but challenging. Front. Neurosci. 12:525. doi: 10.3389/fnins.2018.00525

Dubois, J., and Adolphs, R. (2016). Building a science of individual differences from fMRI. Trends Cogn. Sci. 20, 425-443. doi: 10.1016/j.tics.2016.03.014

Dubois, J., Galdi, P., Paul, L. K., and Adolphs, R. (2018). A distributed brain network predicts general intelligence from resting-state human neuroimaging data. Philos. Trans. R. Soc. Lond. Ser. B Biol. Sci. 373, pii: 20170284. doi: 10.1098/rstb.2017.0284

Edwards, E. S., Burke, K., Booth, J. R., and McNorgan, C. (2018). Dyslexia on a continuum: a complex network approach. PLoS One 13:e0208923. doi 10.1371/journal.pone.0208923

Eickhoff, S., Nichols, T. E., Van Horn, J. D., and Turner, J. A. (2016). Sharing the wealth: neuroimaging data repositories. NeuroImage 124, 1065-1068. doi: 10.1016/j.neuroimage.2015.10.079

Eklund, A., Nichols, T. E., and Knutsson, H. (2016). Cluster failure: why fMRI inferences for spatial extent have inflated false-positive rates. Proc. Natl. Acad. Sci. USA 113, 7900-7905. doi: 10.1073/pnas.1602413113

Elliott, M. L., Knodt, A. R., Cooke, M., Kim, M. J., Melzer, T. R., Keenan, R., et al. (2019). General functional connectivity: shared features of resting-state and task fMRI drive reliable and heritable individual differences in functional brain networks. NeuroImage 189, 516-532. doi: 10.1016/j. neuroimage.2019.01.068

Elsner, B., Kugler, J., Pohl, M., and Mehrholz, J. (2015). Transcranial direct current stimulation (tDCS) for improving aphasia in patients with aphasia after stroke. Cochrane Database Syst. Rev. 5:CD009760. doi: 10.1002/14651858. CD009760.pub3 
Engelhardt, L. E., Roe, M. A., Juranek, J., DeMaster, D., Harden, K. P., Tucker-Drob, E. M., et al. (2017). Children's head motion during fMRI tasks is heritable and stable over time. Dev. Cogn. Neurosci. 25, 58-68. doi: 10.1016/j.dcn.2017.01.011

Esteban, O., Markiewicz, C. J., Blair, R. W., Moodie, C. A., Isik, A. I., Erramuzpe, A., et al. (2019). fMRIPrep: a robust preprocessing pipeline for functional MRI. Nat. Methods 16, 111-116. doi: 10.1038/s41592-018-0235-4

Evans, T. M., Flowers, D. L., Luetje, M. M., Napoliello, E., and Eden, G. F. (2016). Functional neuroanatomy of arithmetic and word reading and its relationship to age. NeuroImage 143, 304-315. doi: 10.1016/j. neuroimage.2016.08.048

Evans, T. M., Kochalka, J., Ngoon, T. J., Wu, S. S., Qin, S., Battista, C., et al. (2015). Brain structural integrity and intrinsic functional connectivity forecast 6 year longitudinal growth in children's numerical abilities. J. Neurosci. 35, 11743-11750. doi: 10.1523/JNEUROSCI.0216-15.2015

Farah, R., and Horowitz-Kraus, T. (2019). Increased functional connectivity within and between cognitive-control networks from early infancy to nine years during story listening. Brain Connect. 9, 285-295. doi: 10.1089/ brain. 2018.0625

Fassbender, C., Mukherjee, P., and Schweitzer, J. B. (2017). Minimizing noise in pediatric task-based functional MRI; adolescents with developmental disabilities and typical development. NeuroImage 149, 338-347. doi: 10.1016/j. neuroimage.2017.01.021

Fatima, Z., Kovacevic, N., Misic, B., and McIntosh, A. R. (2016). Dynamic functional connectivity shapes individual differences in associative learning. Hum. Brain Mapp. 37, 3911-3928. doi: 10.1002/hbm.23285

Feiler, J. B., and Stabio, M. E. (2018). Three pillars of educational neuroscience from three decades of literature. Trends Neurosci. Educ. 13, 17-25. doi: 10.1016/j.tine.2018.11.001

Finn, E. S., Shen, X., Scheinost, D., Rosenberg, M. D., Huang, J., Chun, M. M., et al. (2015). Functional connectome fingerprinting: identifying individuals using patterns of brain connectivity. Nat. Neurosci. 18, 1664-1671. doi: $10.1038 / \mathrm{nn} .4135$

Fiori, V., Kunz, L., Kuhnke, P., Marangolo, P., and Hartwigsen, G. (2018). Transcranial direct current stimulation (tDCS) facilitates verb learning by altering effective connectivity in the healthy brain. NeuroImage 181, 550-559. doi: 10.1016/j.neuroimage.2018.07.040

Fischer-Baum, S., Kook, J. H., Lee, Y., Ramos-Nuñez, A., and Vannucci, M. (2018). Individual differences in the neural and cognitive mechanisms of single word reading. Front. Hum. Neurosci. 12:271. doi: 10.3389/ fnhum.2018.00271

Flöel, A. (2014). tDCS-enhanced motor and cognitive function in neurological diseases. NeuroImage 85, 934-947. doi: 10.1016/j.neuroimage.2013.05.098

Foulkes, L., and Blakemore, S. J. (2018). Studying individual differences in human adolescent brain development. Nat. Neurosci. 21, 315-323. doi: 10.1038/s41593-018-0078-4

Friston, K. J. (2002). Dysfunctional connectivity in schizophrenia. World Psychiatry 1, 66-71.

Friston, K. J. (2009). Modalities, modes, and models in functional neuroimaging. Science 326, 399-403. doi: 10.1126/science.1174521

Friston, K. J. (2011). Functional and effective connectivity: a review. Brain Connect. 1, 13-36. doi: 10.1089/brain.2011.0008

Friston, K. J., Harrison, L., and Penny, W. (2003). Dynamic causal modelling. NeuroImage 19, 1273-1302. doi: 10.1016/S1053-8119(03)00202-7

Friston, K. J., Litvak, V., Oswal, A., Razi, A., Stephan, K. E., van Wijk, B. C., et al. (2016). Bayesian model reduction and empirical Bayes for group (DCM) studies. NeuroImage 128, 413-431. doi: 10.1016/j.neuroimage.2015.11.015

Frith, U. (2011). Brain waves module 2: Neuroscience: Implications for education and lifelong learning. London: Science Policy Centre, The Royal Society.

Fuhrmann, D., Schweizer, S., Leung, J., Griffin, C., and Blakemore, S. J. (2019). The neurocognitive correlates of academic diligence in adolescent girls. Cogn. Neurosci. 10, 88-99. doi: 10.1080/17588928.2018.1504762

Gao, W., Grewen, K., Knickmeyer, R. C., Qiu, A., Salzwedel, A., Lin, W., et al. (2019). A review on neuroimaging studies of genetic and environmental influences on early brain development. NeuroImage 185, 802-812. doi: 10.1016/j.neuroimage.2018.04.032

Geng, F., Redcay, E., and Riggins, T. (2019). The influence of age and performance on hippocampal function and the encoding of contextual information in early childhood. NeuroImage 195, 433-443. doi: 10.1016/j.neuroimage.2019.03.035
Giorgio, A., Watkins, K. E., Chadwick, M., James, S., Winmill, L., Douaud, G., et al. (2010). Longitudinal changes in grey and white matter during adolescence. NeuroImage 49, 94-103. doi: 10.1016/j.neuroimage.2009.08.003

Gomides, M. R. A., Martins, G. A., Alves, I. S., Julio-Costa, A., Jaeger, A., and Haase, V. G. (2018). Heterogeneity of math difficulties and its implications for interventions in multiplication skills. Dement. Neuropsychol. 12, 256-263. doi: 10.1590/1980-57642018dn12-030006

Gonon, F., Bezard, E., and Boraud, T. (2011). Misrepresentation of neuroscience data might give rise to misleading conclusions in the media: the case of attention deficit hyperactivity disorder. PLoS One 6:e14618. doi: 10.1371/journal. pone. 0014618

Gorgolewski, K. J., and Poldrack, R. A. (2016). A practical guide for improving transparency and reproducibility in neuroimaging research. PLoS Biol. 14:e1002506. doi: 10.1371/journal.pbio.1002506

Gorgolewski, K. J., Varoquaux, G., Rivera, G., Schwarz, Y., Ghosh, S. S., Maumet, C., et al. (2015). NeuroVault.org: a web-based repository for collecting and sharing unthresholded statistical maps of the human brain. Front. Neuroinform. 9:8. doi: 10.3389/fninf.2015.00008

Goswami, U. (2004). Neuroscience and education. Br. J. Educ. Psychol. 74, 1-14. doi: $10.1348 / 000709904322848798$

Greene, D. J., Black, K. J., and Schlaggar, B. L. (2016). Considerations for MRI study design and implementation in pediatric and clinical populations. Dev. Cogn. Neurosci. 18, 101-112. doi: 10.1016/j.dcn.2015.12.005

Greene, D. J., Koller, J. M., Hampton, J. M., Wesevich, V., Van, A. N., Nguyen, A. L., et al. (2018). Behavioral interventions for reducing head motion during MRI scans in children. NeuroImage 171, 234-245. doi: 10.1016/j. neuroimage.2018.01.023

Groen, M. A., Whitehouse, A. J., Badcock, N. A., and Bishop, D. V. (2012). Does cerebral lateralization develop? A study using functional transcranial Doppler ultrasound assessing lateralization for language production and visuospatial memory. Brain Behav. 2, 256-269. doi: 10.1002/brb3.56

Haller, S., and Bartsch, A. J. (2009). Pitfalls in fMRI. Eur. Neurol. 19, 2689-2706. doi: 10.1007/s00330-009-1456-9

Halsey, L. G., Curran-Everett, D., Vowler, S. L., and Drummond, G. B. (2015). The fickle P value generates irreproducible results. Nat. Methods 12, 179-185. doi: $10.1038 /$ nmeth.3288

Han, H. (2017). Neural correlates of moral sensitivity and moral judgment associated with brain circuitries of selfhood: a meta-analysis. J. Moral Ed. 46, 97-113. doi: 10.1080/03057240.2016.1262834

Han, H., and Park, J. (2018). Using SPM 12's second-level Bayesian inference procedure for fMRI analysis: practical guidelines for end users. Front. Neuroinform. 12:1. doi: 10.3389/fninf.2018.00001

Han, H., and Park, J. (2019). Bayesian meta-analysis of fMRI image data. Cogn. Neurosci. 10, 66-76. doi: 10.1080/17588928.2019.1570103

Han, H., Soylu, F., and Anchan, D. M. (2019). Connecting levels of analysis in educational neuroscience: a review of multi-level structure of educational neuroscience with concrete examples. Trends Neurosci. Educ. 17:100113. doi: 10.1016/j.tine.2019.100113

Hanke, M., Adelhofer, N., Kottke, D., Iacovella, V., Sengupta, A., Kaule, F. R., et al. (2016). A studyforrest extension, simultaneous fMRI and eye gaze recordings during prolonged natural stimulation. Sci. Data 3:160092. doi: 10.1038/sdata.2016.92

Harvey, J. L., Demetriou, L., McGonigle, J., and Wall, M. B. (2018). A short, robust brain activation control task optimised for pharmacological fMRI studies. PeerJ 6:e5540. doi: 10.7717/peerj.5540

Hasson, U., and Honey, C. J. (2012). Future trends in neuroimaging: neural processes as expressed within real-life contexts. NeuroImage 62, 1272-1278. doi: 10.1016/j.neuroimage.2012.02.004

Hauser, T. U., Rütsche, B., Wurmitzer, K., Brem, S., Ruff, C. C., and Grabner, R. H. (2016). Neurocognitive effects of transcranial direct current stimulation in arithmetic learning and performance: a simultaneous tDCS-fMRI study. Brain Stimul. 9, 850-858. doi: 10.1016/j.brs.2016.07.007

Havlicek, M., Roebroeck, A., Friston, K., Gardumi, A., Ivanov, D., and Uludag, K. (2015). Physiologically informed dynamic causal modeling of fMRI data. NeuroImage 122, 355-372. doi: 10.1016/j.neuroimage.2015.07.078

He, H., Sui, J., Du, Y., Yu, Q., Lin, D., Drevets, W. C., et al. (2017). Co-altered functional networks and brain structure in unmedicated patients with bipolar and major depressive disorders. Brain Struct. Funct. 222, 4051-4064. doi: 10.1007/s00429-017-1451-x 
Hennig, J., Speck, O., Koch, M. A., and Weiller, C. (2003). Functional magnetic resonance imaging: a review of methodological aspects and clinical applications. J. Magn. Reson. Imaging 18, 1-15. doi: 10.1002/jmri.10330

Herting, M. M., Gautam, P., Chen, Z., Mezher, A., and Vetter, N. C. (2018). Testretest reliability of longitudinal task-based fMRI: implications for developmental studies. Dev. Cogn. Neurosci. 33, 17-26. doi: 10.1016/j.dcn.2017.07.001

Herwig, U., Lutz, J., Scherpiet, S., Scheerer, H., Kohlberg, J., Opialla, S., et al. (2019). Training emotion regulation through real-time fMRI neurofeedback of amygdala activity. NeuroImage 184, 687-696. doi: 10.1016/j. neuroimage.2018.09.068

Higgins, I. A., Kundu, S., and Guo, Y. (2018). Integrative Bayesian analysis of brain functional networks incorporating anatomical knowledge. NeuroImage 181, 263-278. doi: 10.1016/j.neuroimage.2018.07.015

Hilger, K., Ekman, M., Fiebach, C. J., and Basten, U. (2017). Intelligence is associated with the modular structure of intrinsic brain networks. Sci. Rep. 7:16088. doi: 10.1038/s41598-017-15795-7

Hong, S. J., Vos de Wael, R., Bethlehem, R. A. I., Lariviere, S., Paquola, C., Valk, S. L., et al. (2019). Atypical functional connectome hierarchy in autism. Nat. Commun. 10:1022. doi: 10.1038/s41467-019-08944-1

Hoogman, M., Muetzel, R., Guimaraes, J. P., Shumskaya, E., Mennes, M., Zwiers, M. P., et al. (2019). Brain imaging of the cortex in ADHD: a coordinated analysis of large-scale clinical and population-based samples. Am. J. Psychiatry 176, 531-542. doi: 10.1176/appi.ajp.2019.18091033

Horowitz-Kraus, T., Toro-Serey, C., and DiFrancesco, M. (2015). Increased resting-state functional connectivity in the Cingulo-Opercular cognitivecontrol network after intervention in children with reading difficulties. PLoS One 10:e0133762. doi: 10.1371/journal.pone.0133762

Houde, O., Rossi, S., Lubin, A., and Joliot, M. (2010). Mapping numerical processing, reading, and executive functions in the developing brain: an fMRI meta-analysis of 52 studies including 842 children. Dev. Sci. 13, 876-885. doi: 10.1111/j.1467-7687.2009.00938.x

Howell, B. R., Styner, M. A., Gao, W., Yap, P. T., Wang, L., Baluyot, K., et al. (2019). The UNC/UMN baby connectome project (BCP): an overview of the study design and protocol development. NeuroImage 185, 891-905. doi: 10.1016/j.neuroimage.2018.03.049

Huber, L., Handwerker, D. A., Jangraw, D. C., Chen, G., Hall, A., Stüber, C., et al. (2017). High-resolution CBV-fMRI allows mapping of laminar activity and connectivity of cortical input and output in human M1. Neuron 96, 1253-1263. doi: 10.1016/j.neuron.2017.11.005

Hykin, J., Moore, R., Duncan, K., Clare, S., Baker, P., Johnson, I., et al. (1999). Fetal brain activity demonstrated by functional magnetic resonance imaging. Lancet 354, 645-646. doi: 10.1016/S0140-6736(99)02901-3

Ikeda, T., Takahashi, T., Hiraishi, H., Saito, D. N., and Kikuchi, M. (2019). Anodal transcranial direct current stimulation induces high gamma-band activity in the left dorsolateral prefrontal cortex during a working memory task: a double-blind, randomized, crossover study. Front. Hum. Neurosci. 13:136. doi: 10.3389/fnhum.2019.00136

Immordino-Yang, M. H., and Gotlieb, R. (2017). Embodied brains, social minds, cultural meaning: integrating neuroscientific and educational research on social-affective development. Am. Educ. Res. J. 54, 344S-367S. doi: 10.3102/0002831216669780

Ioannidis, J. P. (2005). Why most published research findings are false. PLoS Med. 2:e124. doi: 10.1371/journal.pmed.0020124

Jang, C., Knight, E. Q., Pae, C., Park, B., Yoon, S. A., and Park, H. J. (2017). Individuality manifests in the dynamic reconfiguration of large-scale brain networks during movie viewing. Sci. Rep. 7:41414. doi: 10.1038/srep41414

Jezzard, P., and Song, A. W. (1996). Technical foundations and pitfalls of clinical fMRI. NeuroImage 3, S63-S75.

Jokeit, H., Okujava, M., and Woermann, F. G. (2001). Carbamazepine reduces memory induced activation of mesial temporal lobe structures: a pharmacological fMRI-study. BMC Neurol. 1:6. doi: 10.1186/ 1471-2377-1-6

Kajimura, S., Kochiyama, T., Nakai, R., Abe, N., and Nomura, M. (2016). Causal relationship between effective connectivity within the default mode network and mind-wandering regulation and facilitation. NeuroImage 133, 21-30. doi: 10.1016/j.neuroimage.2016.03.009

Keil, B., Alagappan, V., Mareyam, A., McNab, J. A., Fujimoto, K., Tountcheva, V., et al. (2011). Size-optimized 32-channel brain arrays for $3 \mathrm{~T}$ pediatric imaging. Magn. Reson. Med. 66, 1777-1787. doi: 10.1002/mrm.22961
Khan, N. A., and Hillman, C. H. (2014). The relation of childhood physical activity and aerobic fitness to brain function and cognition: a review. Pediatr. Exerc. Sci. 26, 138-146. doi: 10.1123/pes.2013-0125

Khazaee, A., Ebrahimzadeh, A., and Babajani-Feremi, A. (2015). Identifying patients with Alzheimer's disease using resting-state fMRI and graph theory. Clin. Neurophysiol. 126, 2132-2141. doi: 10.1016/j.clinph.2015.02.060

Kherif, F., Josse, G., Seghier, M. L., and Price, C. J. (2009). The main sources of inter-subject variability in neuronal activation for reading aloud. J. Cogn. Neurosci. 21, 654-668. doi: 10.1162/jocn.2009.21084

Kim, H. G., Moon, W. J., Han, J., and Choi, J. W. (2017). Quantification of myelin in children using multiparametric quantitative MRI: a pilot study. Neuroradiology 59, 1043-1051. doi: 10.1007/s00234-017-1889-9

King, K. M., Littlefield, A. K., McCabe, C. J., Mills, K. L., Flournoy, J., and Chassin, L. (2018). Longitudinal modeling in developmental neuroimaging research: common challenges, and solutions from developmental psychology. Dev. Cogn. Neurosci. 33, 54-72. doi: 10.1016/j.dcn.2017.11.009

Kober, S. E., Schweiger, D., Witte, M., Reichert, J. L., Grieshofer, P., Neuper, C., et al. (2015). Specific effects of EEG based neurofeedback training on memory functions in post-stroke victims. J. Neuroeng. Rehabil. 12:107. doi: 10.1186/ s12984-015-0105-6

Konig, C., and van de Schoot, R. (2018). Bayesian statistics in educational research: a look at the current state of affairs. Educ. Rev. 70, 486-509. doi: 10.1080/00131911.2017.1350636

Kopell, N. J., Gritton, H. J., Whittington, M. A., and Kramer, M. A. (2014). Beyond the connectome: the dynome. Neuron 83, 1319-1328. doi: 10.1016/j. neuron.2014.08.016

Kosslyn, S. M., Cacioppo, J. T., Davidson, R. J., Hugdahl, K., Lovallo, W. R., Spiegel, D., et al. (2002). Bridging psychology and biology. The analysis of individuals in groups. Am. Psychol. 57, 341-351. doi: 10.1037/0003-066X.57.5.341

Kotsoni, E., Byrd, D., and Casey, B. J. (2006). Special considerations for functional magnetic resonance imaging of pediatric populations. J. Magn. Reson. Imaging 23, 877-886. doi: 10.1002/jmri.20578

Koyama, M. S., Di Martino, A., Zuo, X. N., Kelly, C., Mennes, M., Jutagir, D. R., et al. (2011). Resting-state functional connectivity indexes reading competence in children and adults. J. Neurosci. 31, 8617-8624. doi: 10.1523/ JNEUROSCI.4865-10.2011

Kozberg, M., and Hillman, E. (2016). Neurovascular coupling and energy metabolism in the developing brain. Prog. Brain Res. 225, 213-242. doi: 10.1016/bs.pbr.2016.02.002

Lahnakoski, J. M., Glerean, E., Salmi, J., Jääskeläinen, I. P., Sams, M., Hari, R., et al. (2012). Naturalistic FMRI mapping reveals superior temporal sulcus as the hub for the distributed brain network for social perception. Front. Hum. Neurosci. 6:233. doi: 10.3389/fnhum.2012.00233

Laird, A. R., Fox, P. M., Eickhoff, S. B., Turner, J. A., Ray, K. L., McKay, D. R., et al. (2011). Behavioral interpretations of intrinsic connectivity networks. J. Cogn. Neurosci. 23, 4022-4037. doi: 10.1162/jocn_a_00077

Larivière, S., Vos de Wael, R., Hong, S. J., Paquola, C., Tavakol, S., Lowe, A. J., et al. (2019). Multiscale structure-function gradients in the neonatal connectome. Cereb. Cortex pii: bhz069. doi: 10.1093/cercor/bhz069 [Epub ahead of print].

Leung, J., Kosinski, P. D., Croal, P. L., and Kassner, A. (2016). Developmental trajectories of cerebrovascular reactivity in healthy children and young adults assessed with magnetic resonance imaging. J. Physiol. 594, 2681-2689. doi: 10.1113/JP271056

LeVan, P., Akin, B., and Hennig, J. (2018). Fast imaging for mapping dynamic networks. NeuroImage 180, 547-558. doi: 10.1016/j.neuroimage.2017.08.029

Li, J., Li, Q., Dai, X., Li, J., and Zhang, X. (2019b). Does pre-scanning training improve the image quality of children receiving magnetic resonance imaging?: a meta-analysis of current studies. Medicine 98:e14323. doi: 10.1097/ MD.0000000000014323

Li, G., Wang, L., Yap, P. T., Wang, F., Wu, Z., Meng, Y., et al. (2019a). Computational neuroanatomy of baby brains: a review. Neurolmage 185, 906-925. doi: 10.1016/j.neuroimage.2018.03.042

Li, R., Yin, S., Zhu, X., Ren, W., Yu, J., Wang, P., et al. (2017). Linking interindividual variability in functional brain connectivity to cognitive ability in elderly individuals. Front. Aging Neurosci. 9:385. doi: 10.3389/fnagi.2017.00385

Li, X., Zhu, D., Jiang, X., Jin, C., Zhang, X., Guo, L., et al. (2014). Dynamic functional connectomics signatures for characterization and differentiation of PTSD patients. Hum. Brain Mapp. 35, 1761-1778. doi: 10.1002/hbm.22290 
Liang, H., and Wang, H. (2017). Structure-function network mapping and its assessment via persistent homology. PLoS Comput. Biol. 13:e1005325. doi: 10.1371/journal.pcbi.1005325

Liew, S. L., Rana, M., Cornelsen, S., Fortunato de Barros Filho, M., Birbaumer, N., Sitaram, R., et al. (2016). Improving motor corticothalamic communication after stroke using real-time fMRI connectivity-based neurofeedback. Neurorehabil. Neural Repair 30, 671-675. doi: $10.1177 / 1545968315619699$

Linhartová, P., Látalová, A., Kóša, B., Kašpárek, T., Schmahl, C., and Paret, C. (2019). fMRI neurofeedback in emotion regulation: a literature review. NeuroImage 193, 75-92. doi: 10.1016/j.neuroimage.2019.03.011

Locke, H. S., and Braver, T. S. (2008). Motivational influences on cognitive control: behavior, brain activation, and individual differences. Cogn. Affect. Behav. Neurosci. 8, 99-112. doi: 10.3758/CABN.8.1.99

Lorca-Puls, D. L., Gajardo-Vidal, A., White, J., Seghier, M. L., Leff, A. P., Green, D. W., et al. (2018). The impact of sample size on the reproducibility of voxel-based lesion-deficit mappings. Neuropsychologia 115, 101-111. doi: 10.1016/j.neuropsychologia.2018.03.014

Lowe, M. J. (2012). The emergence of doing "nothing" as a viable paradigm design. NeuroImage 62, 1146-1151. doi: 10.1016/j.neuroimage.2012.01.014

Lundell, H., Nilsson, M., Dyrby, T. B., Parker, G. J. M., Cristinacce, P. L. H., Zhou, F. L., et al. (2019). Multidimensional diffusion MRI with spectrally modulated gradients reveals unprecedented microstructural detail. Sci. Rep. 9:9026. doi: 10.1038/s41598-019-45235-7

Lyytinen, H., Erskine, J., Hämäläinen, J., Torppa, M., and Ronimus, M. (2015). Dyslexia-early identification and prevention: highlights from the Jyväskylä longitudinal study of dyslexia. Curr. Dev. Disord. Rep. 2, 330-338. doi: 10.1007/s40474-015-0067-1

Madhyastha, T., Peverill, M., Koh, N., McCabe, C., Flournoy, J., Mills, K., et al. (2018). Current methods and limitations for longitudinal fMRI analysis across development. Dev. Cogn. Neurosci. 33, 118-128. doi: 10.1016/j.den.2017.11.006

Maguire, E. A. (2012). Studying the freely-behaving brain with fMRI. NeuroImage 62, 1170-1176. doi: 10.1016/j.neuroimage.2012.01.009

Malinen, S., Hlushchuk, Y., and Hari, R. (2007). Towards natural stimulation in fMRI-issues of data analysis. NeuroImage 35, 131-139. doi: 10.1016/j. neuroimage.2006.11.015

Malins, J. G., Pugh, K. R., Buis, B., Frost, S. J., Hoeft, F., Landi, N., et al. (2018). Individual differences in reading skill are related to trial-by-trial neural activation variability in the reading network. J. Neurosci. 38, 29812989. doi: 10.1523/JNEUROSCI.0907-17.2018

Margari, L., Buttiglione, M., Craig, F., Cristella, A., de Giambattista, C., Matera, E., et al. (2013). Neuropsychopathological comorbidities in learning disorders. BMC Neurol. 13:198. doi: 10.1186/1471-2377-13-198

Marquand, A. F., Kia, S. M., Zabihi, M., Wolfers, T., Buitelaar, J. K., and Beckmann, C. F. (2019). Conceptualizing mental disorders as deviations from normative functioning. Mol. Psychiatry 24, 1415-1424. doi: 10.1038/ s41380-019-0441-1

Mathiak, K., and Weber, R. (2006). Toward brain correlates of natural behavior: fMRI during violent video games. Hum. Brain Mapp. 27, 948-956. doi: 10.1002/hbm.20234

Mattar, M. G., Wymbs, N. F., Bock, A. S., Aguirre, G. K., Grafton, S. T., and Bassett, D. S. (2018). Predicting future learning from baseline network architecture. NeuroImage 172, 107-117. doi: 10.1016/j.neuroimage. 2018.01.037

Mayer, R. E. (2017). How can brain research inform academic learning and instruction? Educ. Psychol. Rev. 29, 835-846. doi: 10.1007/s10648-016-9391-1

McColgan, P., Razi, A., Gregory, S., Seunarine, K. K., Durr, A., Roos, A. C. R., et al. (2017). Structural and functional brain network correlates of depressive symptoms in premanifest Huntington's disease. Hum. Brain Mapp. 38, 2819-2829. doi: 10.1002/hbm.23527

McFarland, D. J. (2017). How neuroscience can inform the study of individual differences in cognitive abilities. Rev. Neurosci. 28, 343-362. doi: 10.1515/ revneuro-2016-0073

Meier, J., Tewarie, P., Hillebrand, A., Douw, L., van Dijk, B. W., Stufflebeam, S. M., et al. (2016). A mapping between structural and functional brain networks. Brain Connect. 6, 298-311. doi: 10.1089/brain.2015.0408

Mennes, M., Kelly, C., Zuo, X. N., Di Martino, A., Biswal, B. B., Castellanos, F. X., et al. (2010). Inter-individual differences in resting-state functional connectivity predict task-induced BOLD activity. NeuroImage 50, 1690-1701. doi: 10.1016/j.neuroimage.2010.01.002

Mennes, M., Zuo, X. N., Kelly, C., Di Martino, A., Zang, Y. F., Biswal, B., et al. (2011). Linking inter-individual differences in neural activation and behavior to intrinsic brain dynamics. NeuroImage 54, 2950-2959. doi: 10.1016/j. neuroimage.2010.10.046

Mill, R. D., Ito, T., and Cole, M. W. (2017). From connectome to cognition: the search for mechanism in human functional brain networks. NeuroImage 160, 124-139. doi: 10.1016/j.neuroimage.2017.01.060

Miranda-Dominguez, O., Mills, B. D., Carpenter, S. D., Grant, K. A., Kroenke, C. D., Nigg, J. T., et al. (2014). Connectotyping: model based fingerprinting of the functional connectome. PLoS One 9:e111048. doi: 10.1371/journal. pone. 0111048

Mišić, B., and Sporns, O. (2016). From regions to connections and networks: new bridges between brain and behavior. Curr. Opin. Neurobiol. 40, 1-7. doi: 10.1016/j.conb.2016.05.003

Moraczewski, D., Chen, G., and Redcay, E. (2018). Inter-subject synchrony as an index of functional specialization in early childhood. Sci. Rep. 8:2252. doi: 10.1038/s41598-018-20600-0

Morken, F., Helland, T., Hugdahl, K., and Specht, K. (2017). Reading in dyslexia across literacy development: a longitudinal study of effective connectivity. NeuroImage 144, 92-100. doi: 10.1016/j.neuroimage.2016.09.060

Mueller, S. G., Weiner, M. W., Thal, L. J., Petersen, R. C., Jack, C. R., Jagust, W., et al. (2005). Ways toward an early diagnosis in Alzheimer's disease: the Alzheimer's disease neuroimaging initiative (ADNI). Alzheimers Dement. 1, 55-66. doi: 10.1016/j.jalz.2005.06.003

Muller, V. I., Cieslik, E. C., Laird, A. R., Fox, P. T., Radua, J., Mataix-Cols, D., et al. (2018). Ten simple rules for neuroimaging meta-analysis. Neurosci. Biobehav. Rev. 84, 151-161. doi: 10.1016/j.neubiorev.2017.11.012

Murdaugh, D. L., Maximo, J. O., and Kana, R. K. (2015). Changes in intrinsic connectivity of the brain's reading network following intervention in children with autism. Hum. Brain Mapp. 36, 2965-2979. doi: 10.1002/hbm.22821

Neville, H. J., Stevens, C., Pakulak, E., Bell, T. A., Fanning, J., Klein, S., et al. (2013). Family-based training program improves brain function, cognition, and behavior in lower socioeconomic status preschoolers. Proc. Natl. Acad. Sci. USA 110, 12138-12143. doi: 10.1073/pnas.1304437110

$\mathrm{Ng}$, B. (2018). The neuroscience of growth mindset and intrinsic motivation. Brain Sci. 8:20. doi: 10.3390/brainsci8020020

Nichols, T. E., Das, S., Eickhoff, S. B., Evans, A. C., Glatard, T., Hanke, M., et al. (2017). Best practices in data analysis and sharing in neuroimaging using MRI. Nat. Neurosci. 20, 299-303. doi: 10.1038/nn.4500

Nieder, A. (2016). The neuronal code for number. Nat. Rev. Neurosci. 17, 366-382. doi: 10.1038/nrn.2016.40

Nieuwenhuis, S., Forstmann, B. U., and Wagenmakers, E. J. (2011). Erroneous analyses of interactions in neuroscience: a problem of significance. Nat. Neurosci. 14, 1105-1107. doi: 10.1038/nn.2886

Nissen, S. B., Magidson, T., Gross, K., and Bergstrom, C. T. (2016). Publication bias and the canonization of false facts. elife 5:e21451. doi: 10.7554/ eLife. 21451

Nora, A., Karvonen, L., Renvall, H., Parviainen, T., Kim, J. Y., Service, E., et al. (2017). Children show right-lateralized effects of spoken word-form learning. PLoS One 12:e0171034. doi: 10.1371/journal.pone.0171034

Nugiel, T., Roe, M. A., Taylor, W. P., Cirino, P. T., Vaughn, S. R., Fletcher, J. M., et al. (2019). Brain activity in struggling readers before intervention relates to future reading gains. Cortex 111, 286-302. doi: 10.1016/j.cortex.2018.11.009

Ofen, N., Tang, L., Yu, Q., and Johnson, E. L. (2019). Memory and the developing brain: from description to explanation with innovation in methods. Dev. Cogn. Neurosci. 36:100613. doi: 10.1016/j.den.2018.12.011

Ofori, E., Du, G., Babcock, D., Huang, X., and Vaillancourt, D. E. (2016). Parkinson's disease biomarkers program brain imaging repository. NeuroImage, 124, 1120-1124. doi: 10.1016/j.neuroimage.2015.05.005

Osorio, A. A. C., and Brunoni, A. R. (2019). Transcranial direct current stimulation in children with autism spectrum disorder: a systematic scoping review. Dev. Med. Child Neurol. 61, 298-304. doi: 10.1111/dmcn.14104

Ozernov-Palchik, O., Yu, X., Wang, Y., and Gaab, N. (2016). Lessons to be learned: how a comprehensive neurobiological framework of atypical reading development can inform educational practice. Curr. Opin. Behav. Sci. 10, 45-58. doi: 10.1016/j.cobeha.2016.05.006 
Parsey, R. (2018). The importance of publishing negative findings in science. Biol. Psychiatry 84:550. doi: 10.1016/j.biopsych.2018.08.010

Payakachat, N., Tilford, J. M., and Ungar, W. J. (2016). National database for autism research (NDAR): big data opportunities for health services research and health technology assessment. PharmacoEconomics 34, 127-138. doi: 10.1007/s40273-015-0331-6

Peelen, M. V., and Kastner, S. (2014). Attention in the real world: toward understanding its neural basis. Trends Cogn. Sci. 18, 242-250. doi: 10.1016/j. tics.2014.02.004

Pessoa, L., and McMenamin, B. (2017). Dynamic networks in the emotional brain. Neuroscientist 23, 383-396. doi: 10.1177/1073858416671936

Petitto, L. A., and Dunbar, K. N. (2009). Educational neuroscience: new discoveries from bilingual brains, scientific brains, and the educated mind. Mind Brain Educ. 3, 185-197. doi: 10.1111/j.1751-228X.2009.01069.x

Pfeffer, C., and Olsen, B. R. (2002). Editorial: journal of negative results in biomedicine. J. Neg. Res. Biomed. 1, 1-2. doi: 10.1186/1477-5751-1-2

Pleisch, G., Karipidis, I. I., Brauchli, C., Röthlisberger, M., Hofstetter, C., Stämpfli, P., et al. (2019). Emerging neural specialization of the ventral occipitotemporal cortex to characters through phonological association learning in preschool children. NeuroImage 189, 813-831. doi: 10.1016/j. neuroimage.2019.01.046

Polak, D., Cauley, S., Huang, S. Y., Longo, M. G., Conklin, J., Bilgic, B., et al. (2019). Highly-accelerated volumetric brain examination using optimized waveCAIPI encoding. J. Magn. Reson. Imaging 50, 961-974. doi: 10.1002/jmri.26678

Poldrack, R. A. (2006). Can cognitive processes be inferred from neuroimaging data? Trends Cogn. Sci. 10, 59-63. doi: 10.1016/j.tics.2005.12.004

Poldrack, R. A., Barch, D. M., Mitchell, J. P., Wager, T. D., Wagner, A. D., Devlin, J. T., et al. (2013). Toward open sharing of task-based fMRI data: the OpenfMRI project. Front. Neuroinform. 7:12. doi: 10.3389/ fninf.2013.00012

Pollack, C., Luk, G., and Christodoulou, J. A. (2015). A meta-analysis of functional reading systems in typically developing and struggling readers across different alphabetic languages. Front. Psychol. 6:191. doi: 10.3389/ fpsyg.2015.00191

Price, C. J., and Friston, K. J. (2002). Degeneracy and cognitive anatomy. Trends Cogn. Sci. 6, 416-421. doi: 10.1016/S1364-6613(02)01976-9

Price, C. J., Hope, T. M., and Seghier, M. L. (2017). Ten problems and solutions when predicting individual outcome from lesion site after stroke. NeuroImage 145, 200-208. doi: 10.1016/j.neuroimage.2016.08.006

Raichle, M. E., MacLeod, A. M., Snyder, A. Z., Powers, W. J., Gusnard, D. A., and Shulman, G. L. (2001). A default mode of brain function. Proc. Natl. Acad. Sci. USA 98, 676-682. doi: 10.1073/pnas.98.2.676

Razi, A., Seghier, M. L., Zhou, Y., McColgan, P., Zeidman, P., Park, H. J., et al. (2017). Large-scale DCMs for resting state fMRI. Network Neurosci. 1, 222-241. doi: 10.1162/NETN_a_00015

Reid, L. B., Boyd, R. N., Cunnington, R., and Rose, S. E. (2016). Interpreting intervention induced neuroplasticity with fMRI: the case for multimodal imaging strategies. Neural Plast. 2016:2643491. doi: 10.1155/2016/2643491

Reijneveld, J. C., Ponten, S. C., Berendse, H. W., and Stam, C. J. (2007). The application of graph theoretical analysis to complex networks in the brain. Clin. Neurophysiol. 118, 2317-2331. doi: 10.1016/j.clinph.2007.08.010

Reinkensmeyer, D. J., Burdet, E., Casadio, M., Krakauer, J. W., Kwakkel, G., Lang, C. E., et al. (2016). Computational neurorehabilitation: modeling plasticity and learning to predict recovery. J. Neuroeng. Rehabil. 13:42. doi: 10.1186/s12984-016-0148-3

Renton, T., Tibbles, A., and Topolovec-Vranic, J. (2017). Neurofeedback as a form of cognitive rehabilitation therapy following stroke: a systematic review. PLoS One 12:e0177290. doi: 10.1371/journal.pone.0177290

Richards, T. L., Berninger, V. W., Yagle, K. J., Abbott, R. D., and Peterson, D. J. (2017). Changes in DTI diffusivity and fMRI connectivity cluster coefficients for students with and without specific learning disabilities in written language: brain's response to writing instruction. J. Nat. Sci. 3:e350.

Richardson, H., Lisandrelli, G., Riobueno-Naylor, A., and Saxe, R. (2018). Development of the social brain from age three to twelve years. Nat. Commun. 9:1027. doi: 10.1038/s41467-018-03399-2

Rigoux, L., and Daunizeau, J. (2015). Dynamic causal modelling of brainbehaviour relationships. NeuroImage 117, 202-221. doi: 10.1016/j. neuroimage.2015.05.041
Rizzolatti, G., Fabbri-Destro, M., Caruana, F., and Avanzini, P. (2018). System neuroscience: past, present, and future. CNS Neurosci. Ther. 24, 685-993. doi: $10.1111 /$ cns. 12997

Roalf, D. R., and Gur, R. C. (2017). Functional brain imaging in neuropsychology over the past 25 years. Neuropsychology 31, 954-971. doi: 10.1037/neu0000426

Rosa, M. J., Bestmann, S., Harrison, L., and Penny, W. (2010). Bayesian model selection maps for group studies. NeuroImage 49, 217-224. doi: 10.1016/j. neuroimage.2009.08.051

Rosen, A. C. (2009). Functional magnetic resonance imaging (FMRI) in the classroom. Am. J. Bioeth. 9, 30-31. doi: 10.1080/15265160802617936

Rosenberg, M. D., Finn, E. S., Scheinost, D., Constable, R. T., and Chun, M. M. (2017). Characterizing attention with predictive network models. Trends Cogn. Sci. 21, 290-302. doi: 10.1016/j.tics.2017.01.011

Rosenblau, G., Kliemann, D., Lemme, B., Walter, H., Heekeren, H. R., and Dziobek, I. (2016). The role of the amygdala in naturalistic mentalising in typical development and in autism spectrum disorder. Br. J. Psychiatry 208, 556-564. doi: 10.1192/bjp.bp.114.159269

Rubia, K., Criaud, M., Wulff, M., Alegria, A., Brinson, H., Barker, G., et al. (2019). Functional connectivity changes associated with fMRI neurofeedback of right inferior frontal cortex in adolescents with ADHD. NeuroImage 188, 43-58. doi: 10.1016/j.neuroimage.2018.11.055

Rubinov, M., and Sporns, O. (2010). Complex network measures of brain connectivity: uses and interpretations. NeuroImage 52, 1059-1069. doi: 10.1016/j.neuroimage.2009.10.003

Sala-Llonch, R., Peña-Gómez, C., Arenaza-Urquijo, E. M., Vidal-Piñeiro, D., Bargalló, N., Junqué, C., et al. (2012). Brain connectivity during resting state and subsequent working memory task predicts behavioural performance. Cortex 48, 1187-1196. doi: 10.1016/j.cortex.2011.07.006

Satterthwaite, T. D., Ciric, R., Roalf, D. R., Davatzikos, C., Bassett, D. S., and Wolf, D. H. (2019). Motion artifact in studies of functional connectivity: characteristics and mitigation strategies. Hum. Brain Mapp. 40, 2033-2051. doi: $10.1002 / \mathrm{hbm} .23665$

Satterthwaite, T. D., Connolly, J. J., Ruparel, K., Calkins, M. E., Jackson, C., Elliott, M. A., et al. (2016). The philadelphia neurodevelopmental cohort: a publicly available resource for the study of normal and abnormal brain development in youth. NeuroImage 124, 1115-1119. doi: 10.1016/j.neuroimage.2015.03.056

Satterthwaite, T. D., Wolf, D. H., Loughead, J., Ruparel, K., Elliott, M. A., Hakonarson, H., et al. (2012). Impact of in-scanner head motion on multiple measures of functional connectivity: relevance for studies of neurodevelopment in youth. NeuroImage 60, 623-632. doi: 10.1016/j.neuroimage.2011.12.063

Scheinost, D., Noble, S., Horien, C., Greene, A. S., Lake, E. M., Salehi, M., et al. (2019). Ten simple rules for predictive modeling of individual differences in neuroimaging. NeuroImage 193, 35-45. doi: 10.1016/j.neuroimage.2019.02.057

Schönenberg, M., Wiedemann, E., Schneidt, A., Scheeff, J., Logemann, A., Keune, P. M., et al. (2017). Neurofeedback, sham neurofeedback, and cognitivebehavioural group therapy in adults with attention-deficit hyperactivity disorder: a triple-blind, randomised, controlled trial. Lancet Psychiatry 4, 673-684. doi: 10.1016/S2215-0366(17)30291-2

Schooler, J. (2011). Unpublished results hide the decline effect. Nature 470:437. doi: $10.1038 / 470437$ a

Schwartz, C., Barican, J. L., Yung, D., Zheng, Y., and Waddell, C. (2019). Six decades of preventing and treating childhood anxiety disorders: a systematic review and meta-analysis to inform policy and practice. Evid. Based Ment. Health 22, 103-110. doi: 10.1136/ebmental-2019-300096

Sebastian, R., Saxena, S., Tsapkini, K., Faria, A. V., Long, C., Wright, A., et al. (2017). Cerebellar tDCS: a novel approach to augment language treatment post-stroke. Front. Hum. Neurosci. 10:695. doi: 10.3389/fnhum.2016.00695

Seghier, M. L., Lazeyras, F., and Huppi, P. S. (2006). Functional MRI of the newborn. Sem. Fetal Neonatal. Med. 11, 479-488. doi: 10.1016/j.siny.2006.07.007

Seghier, M. L., Lee, H. L., Schofield, T., Ellis, C. L., and Price, C. J. (2008). Inter-subject variability in the use of two different neuronal networks for reading aloud familiar words. NeuroImage 42, 1226-1236. doi: 10.1016/j. neuroimage.2008.05.029

Seghier, M. L., and Price, C. J. (2009). Dissociating functional brain networks by decoding the between-subject variability. NeuroImage 45, 349-359. doi: 10.1016/j.neuroimage.2008.12.017

Seghier, M. L., and Price, C. J. (2018). Interpreting and utilising intersubject variability in brain function. Trends Cogn. Sci. 22, 517-530. doi: 10.1016/j.tics.2018.03.003 
Seghier, M. L., Zeidman, P., Neufeld, N. H., Leff, A. P., and Price, C. J. (2010). Identifying abnormal connectivity in patients using dynamic causal modelling of fMRI responses. Front. Sys. Neurosci. 4:142. doi: 10.3389/fnsys.2010.00142

Sigman, M., Peña, M., Goldin, A. P., and Ribeiro, S. (2014). Neuroscience and education: prime time to build the bridge. Nat. Neurosci. 17, 497-502. doi: 10.1038/nn.3672

Silva, G., and Citterio, A. (2017). Hemispheric asymmetries in dorsal language pathway white-matter tracts: a magnetic resonance imaging tractography and functional magnetic resonance imaging study. Neuroradiol. J. 30, 470476. doi: 10.1177/1971400917720829

Sitaram, R., Ros, T., Stoeckel, L., Haller, S., Scharnowski, F., Lewis-Peacock, J., et al. (2017). Closed-loop brain training: the science of neurofeedback. Nat. Rev. Neurosci. 18, 86-100. doi: 10.1038/nrn.2016.164

Skeide, M. A., Evans, T. M., Mei, E. Z., Abrams, D. A., and Menon, V. (2018). Neural signatures of co-occurring reading and mathematical difficulties. Dev. Sci. 21:e12680. doi: 10.1111/desc.12680

Slater, D. A., Melie-Garcia, L., Preisig, M., Kherif, F., Lutti, A., and Draganski, B. (2019). Evolution of white matter tract microstructure across the life span. Hum. Brain Mapp. 40, 2252-2268. doi: 10.1002/hbm. 24522

Smallwood, J., Fishman, D. J., and Schooler, J. W. (2007). Counting the cost of an absent mind: mind wandering as an underrecognized influence on educational performance. Psychon. Bull. Rev. 14, 230-236. doi: 10.3758/ BF03194057

Smallwood, J., and Schooler, J. W. (2015). The science of mind wandering: empirically navigating the stream of consciousness. Annu. Rev. Psychol. 66, 487-518. doi: 10.1146/annurev-psych-010814-015331

Smith, G. J., Booth, J. R., and McNorgan, C. (2018). Longitudinal task-related functional connectivity changes predict Reading development. Front. Psychol. 9:1754. doi: 10.3389/fpsyg.2018.01754

Soares, J. M., Magalhaes, R., Moreira, P. S., Sousa, A., Ganz, E., Sampaio, A., et al. (2016). A Hitchhiker's guide to functional magnetic resonance imaging. Front. Neurosci. 10:515. doi: 10.3389/fnins.2016.00515

Soch, J., and Allefeld, C. (2018). MACS - a new SPM toolbox for model assessment, comparison and selection. J. Neurosci. Methods 306, 19-31. doi: 10.1016/j.jneumeth.2018.05.017

Song, J., Birn, R. M., Boly, M., Meier, T. B., Nair, V. A., Meyerand, M. E., et al. (2014). Age-related reorganizational changes in modularity and functional connectivity of human brain networks. Brain Connect. 4, 662-676. doi: 10.1089/brain.2014.0286

Sousa, D. A. (2010). Mind, brain and education: Neuroscience implications for the classroom. Bloomington: Solution Tree Press.

Sreedharan, R. M., Menon, A. C., James, J. S., Kesavadas, C., and Thomas, S. V. (2015). Arcuate fasciculus laterality by diffusion tensor imaging correlates with language laterality by functional MRI in preadolescent children. Neuroradiology 57, 291-297. doi: 10.1007/s00234-014-1469-1

Supekar, K., Swigart, A. G., Tenison, C., Jolles, D. D., Rosenberg-Lee, M., Fuchs, L., et al. (2013). Neural predictors of individual differences in response to math tutoring in primary-grade school children. Proc. Natl. Acad. Sci. USA 110, 8230-8235. doi: 10.1073/pnas.1222154110

Szaflarski, J. P., Holland, S. K., Schmithorst, V. J., and Byars, A. W. (2006). fMRI study of language lateralization in children and adults. Hum. Brain Mapp. 27, 202-212. doi: 10.1002/hbm.20177

Szeszak, S., Man, R., Love, A., Langmack, G., Wharrad, H., and Dineen, R. A. (2016). Animated educational video to prepare children for MRI without sedation: evaluation of the appeal and value. Pediatr. Radiol. 46, 1744-1750. doi: 10.1007/s00247-016-3661-4

Takashima, A., Bakker-Marshall, I., van Hell, J. G., McQueen, J. M., and Janzen, G. (2019). Neural correlates of word learning in children. Dev. Cogn. Neurosci. 37:100649. doi: 10.1016/j.dcn.2019.100649

Telzer, E. H., McCormick, E. M., Peters, S., Cosme, D., Pfeifer, J. H., and van Duijvenvoorde, A. C. K. (2018). Methodological considerations for developmental longitudinal fMRI research. Dev. Cogn. Neurosci. 33, 149-160. doi: 10.1016/j.dcn.2018.02.004

Thibault, R. T., MacPherson, A., Lifshitz, M., Roth, R. R., and Raz, A. (2018). Neurofeedback with fMRI: a critical systematic review. NeuroImage 172, 786-807. doi: 10.1016/j.neuroimage.2017.12.071

Thieba, C., Long, X., Dewey, D., and Lebel, C. (2019). Young children in different linguistic environments: a multimodal neuroimaging study of the inferior frontal gyrus. Brain Cogn. 134, 71-79. doi: 10.1016/j. bandc.2018.05.009

Thiel, A., and Zumbansen, A. (2016). The pathophysiology of post-stroke aphasia: a network approach. Restor. Neurol. Neurosci. 34, 507-518. doi: 10.3233/ RNN-150632

Thomas, M. S. C., Ansari, D., and Knowland, V. C. P. (2019a). Annual research review: educational neuroscience: progress and prospects. J. Child Psychol. Psychiatry 60, 477-492. doi: 10.1111/jcpp.12973

Thomas, M. S. C., Fedor, A., Davis, R., Yang, J., Alireza, H., Charman, T., et al. (2019b). Computational modeling of interventions for developmental disorders. Psychol. Rev. 126, 693-726. doi: 10.1037/rev0000151

Thompson, P. M., Andreassen, O. A., Arias-Vasquez, A., Bearden, C. E., Boedhoe, P. S., Brouwer, R. M., et al. (2017). ENIGMA and the individual: predicting factors that affect the brain in 35 countries worldwide. NeuroImage 145, 389-408. doi: 10.1016/j.neuroimage.2015.11.057

Thompson-Schill, S. L., Braver, T. S., and Jonides, J. (2005). Individual differences. Cogn. Affect. Behav. Neurosci. 5, 115-116. doi: 10.3758/CABN.5.2.115

Thulborn, K. R., Davis, D., Erb, P., Strorwas, M., and Sweeney, J. A. (1996). Clinical fMRI: implementation and experience. NeuroImage 4, S101-S107. doi: 10.1006/nimg.1996.0060

Tobyne, S. M., Somers, D. C., Brissenden, J. A., Michalka, S. W., Noyce, A. L., and Osher, D. E. (2018). Prediction of individualized task activation in sensory modality-selective frontal cortex with 'connectome fingerprinting'. NeuroImage 183, 173-185. doi: 10.1016/j.neuroimage.2018.08.007

Todd, J. J., and Marois, R. (2005). Posterior parietal cortex activity predicts individual differences in visual short-term memory capacity. Cogn. Affect. Behav. Neurosci. 5, 144-155. doi: 10.3758/CABN.5.2.144

Turner, R. (2016). Uses, misuses, new uses and fundamental limitations of magnetic resonance imaging in cognitive science. Philos. Trans. R. Soc. Lond. Ser. B Biol. Sci. 371:20150349. doi: 10.1098/rstb.2015.0349

Turner, R., and Geyer, S. (2014). Comparing like with like: the power of knowing where you are. Brain Connect. 4, 547-557. doi: 10.1089/brain.2014.0261

Turner, B. O., Paul, E. J., Miller, M. B., and Barbey, A. K. (2018). Small sample sizes reduce the replicability of task-based fMRI studies. Commun. Biol. 1:62. doi: 10.1038/s42003-018-0073-Z

Ugurbil, K. (2018). What is feasible with imaging human brain function and connectivity using functional magnetic resonance imaging. Philos. Trans. $R$. Soc. Lond. Ser. B Biol. Sci. 371, pii: 20150361. doi: 10.1098/rstb.2015.0361

Vanderwal, T., Eilbott, J., and Castellanos, F. X. (2019). Movies in the magnet: naturalistic paradigms in developmental functional neuroimaging. Dev. Cogn. Neurosci. 36:100600. doi: 10.1016/j.dcn.2018.10.004

Vanderwal, T., Kelly, C., Eilbott, J., Mayes, L. C., and Castellanos, F. X. (2015). Inscapes: a movie paradigm to improve compliance in functional magnetic resonance imaging. NeuroImage 122:222. doi: 10.1016/j.neuroimage.2015.07.069

Vasta, R., Cutini, S., Cerasa, A., Gramigna, V., Olivadese, G., Arabia, G., et al. (2018). Physiological aging influence on brain hemodynamic activity during task-switching: a fNIRS study. Front. Aging Neurosci. 9:433. doi: 10.3389/ fnagi.2017.00433

Vega-Pons, S., Olivetti, E., Avesani, P., Dodero, L., Gozzi, A., and Bifone, A. (2017). Differential effects of brain disorders on structural and functional connectivity. Front. Neurosci. 10:605. doi: 10.3389/fnins.2016.00605

Vetter, N. C., Steding, J., Jurk, S., Ripke, S., Mennigen, E., and Smolka, M. N. (2017). Reliability in adolescent fMRI within two years - a comparison of three tasks. Sci. Rep. 7:2287. doi: 10.1038/s41598-017-02334-7

Wagenmakers, E. J., Marsman, M., Jamil, T., Ly, A., Verhagen, J., Love, J., et al. (2018). Bayesian inference for psychology. Part I: theoretical advantages and practical ramifications. Psychon. Bull. Rev. 25, 35-57. doi: 10.3758/ s13423-017-1343-3

Wagenmakers, E. J., Morey, R. D., and Lee, M. D. (2016). Bayesian benefits for the pragmatic researcher. Curr. Direct Psychol. Sci. 25, 169-176. doi: $10.1177 / 0963721416643289$

Wager, T. D., Jonides, J., Smith, E. E., and Nichols, T. E. (2005). Toward a taxonomy of attention shifting: individual differences in fMRI during multiple shift types. Cogn. Affect. Behav. Neurosci. 5, 127-143. doi: 10.3758/ CABN.5.2.127

Waitayawinyu, P., and Wankan, P. (2016). The success of MRI without sedations in 6-15 years old pediatric patients after watching MRI introductory video. J. Med. Assoc. Thail. 99, 596-601. 
Wandell, B. A., and Le, R. K. (2017). Diagnosing the neural circuitry of reading. Neuron 96, 298-311. doi: 10.1016/j.neuron.2017.08.007

Wang, L., Alpert, K. I., Calhoun, V. D., Cobia, D. J., Keator, D. B., King, M. D., et al. (2016). SchizConnect: mediating neuroimaging databases on schizophrenia and related disorders for large-scale integration. NeuroImage 124, 1155-1167. doi: 10.1016/j.neuroimage.2015.06.065

Wang, M. Y., Luan, P., Zhang, J., Xiang, Y. T., Niu, H., and Yuan, Z. (2018). Concurrent mapping of brain activation from multiple subjects during social interaction by hyperscanning: a mini-review. Quant. Imaging Med. Surg. 8, 819-837. doi: 10.21037/qims.2018.09.07

Wang, X., Yang, J., Yang, J., Mencl, W. E., Shu, H., and Zevin, J. D. (2015). Language differences in the brain network for reading in naturalistic story reading and lexical decision. PLoS One 10:e0124388. doi: 10.1371/journal. pone. 0146050

Watanabe, T., Sasaki, Y., Shibata, K., and Kawato, M. (2017). Advances in fMRI real-time neurofeedback. Trends Cogn. Sci. 21, 997-1010. doi: 10.1016/j. tics.2017.09.010

Weisberg, D. S., Keil, F. C., Goodstein, J., Rawson, E., and Gray, J. R. (2008). The seductive allure of neuroscience explanations. J. Cogn. Neurosci. 20, 470-477. doi: 10.1162/jocn.2008.20040

Wilke, M., Groeschel, S., Lorenzen, A., Rona, S., Schuhmann, M. U., Ernemann, U., et al. (2018). Clinical application of advanced MR methods in children: points to consider. Ann. Clin. Transl. Neurol. 5, 1434-1455. doi: 10.1002/acn3.658

Willcutt, E. G., McGrath, L. M., Pennington, B. F., Keenan, J. M., DeFries, J. C., Olson, R. K., et al. (2019). Understanding comorbidity between specific learning disabilities. New Dir. Child Adolesc. Dev. 2019, 91-109. doi: 10.1002/ cad.20291

Winkler, S. A., Corea, J., Lechêne, B., O’Brien, K., Bonanni, J. R., Chaudhari, A., et al. (2019). Evaluation of a flexible 12-channel screen-printed pediatric MRI coil. Radiology 291, 180-185. doi: 10.1148/radiol.2019181883

Wise Younger, J., Tucker-Drob, E., and Booth, J. R. (2017). Longitudinal changes in reading network connectivity related to skill improvement. NeuroImage 158, 90-98. doi: 10.1016/j.neuroimage.2017.06.044

Woo, C. W., Krishnan, A., and Wager, T. D. (2014). Cluster-extent based thresholding in fMRI analyses: pitfalls and recommendations. NeuroImage 91, 412-419. doi: 10.1016/j.neuroimage.2013.12.058

Wu, C., Honarmand, A. R., Schnell, S., Kuhn, R., Schoeneman, S. E., Ansari, S. A., et al. (2016). Age-related changes of normal cerebral and cardiac blood flow in children and adults aged 7 months to 61 years. J. Am. Heart Assoc. 5:e002657. doi: 10.1161/JAHA.115.002657

Wu, Z. M., Llera, A., Hoogman, M., Cao, Q. J., Zwiers, M. P., Bralten, J., et al. (2019). Linked anatomical and functional brain alterations in children with attention-deficit/hyperactivity disorder. Neuroimage Clin. 23:101851. doi: 10.1016/j.nicl.2019.101851

Xue, H., Zhao, L., Wang, Y., Dong, Q., Chen, C., and Xue, G. (2017). Anodal transcranial direct current stimulation over the left temporoparietal cortex facilitates assembled phonology. Trends Neurosci. Educ. 8-9, 10-17. doi: 10.1016/j.tine.2017.08.001

Yahata, N., Morimoto, J., Hashimoto, R., Lisi, G., Shibata, K., Kawakubo, Y., et al. (2016). A small number of abnormal brain connections predicts adult autism spectrum disorder. Nat. Commun. 7:11254. doi: 10.1038/ ncomms 11254
Yaple, Z., and Arsalidou, M. (2018). N-back working memory task: metaanalysis of normative fMRI studies with children. Child Dev. 89, 2010-2022. doi: $10.1111 /$ cdev. 13080

Yarkoni, T., Poldrack, R. A., Nichols, T. E., Van Essen, D. C., and Wager, T. D. (2011). Large-scale automated synthesis of human functional neuroimaging data. Nat. Methods 8, 665-670. doi: 10.1038/nmeth.1635

Yourganov, G., Schmah, T., Small, S. L., Rasmussen, P. M., and Strother, S. C. (2010). Functional connectivity metrics during stroke recovery. Arch. Ital. Biol. 148, 259-270.

Yu, X., Raney, T., Perdue, M. V., Zuk, J., Ozernov-Palchik, O., Becker, B. L. C., et al. (2018). Emergence of the neural network underlying phonological processing from the prereading to the emergent reading stage: a longitudinal study. Hum. Brain Mapp. 39, 2047-2063. doi: 10.1002/hbm.23985

Zaitsev, M., Akin, B., LeVan, P., and Knowles, B. R. (2017). Prospective motion correction in functional MRI. NeuroImage 154, 33-42. doi: 10.1016/j. neuroimage.2016.11.014

Zeidman, P., Jafarian, A., Seghier, M. L., Litvak, V., Cagnan, H., Price, C. J., et al. (2019). A guide to group effective connectivity analysis, part 2: second level analysis with PEB. NeuroImage 200, 12-25. doi: 10.1016/j. neuroimage.2019.06.032

Zhan, C., Liu, Y., Wu, K., Gao, Y., and Li, X. (2017). Structural and functional abnormalities in children with attention-deficit/hyperactivity disorder: a focus on subgenual anterior cingulate cortex. Brain Connect. 7, 106-114. doi: 10.1089/brain.2016.0444

Zhang, H., Shen, D., and Lin, W. (2019). Resting-state functional MRI studies on infant brains: a decade of gap-filling efforts. NeuroImage 185, 664-684. doi: 10.1016/j.neuroimage.2018.07.004

Zhao, Q., Kwon, D., Müller-Oehring, E. M., Le Berre, A. P., Pfefferbaum, A., Sullivan, E. V., et al. (2019). Longitudinally consistent estimates of intrinsic functional networks. Hum. Brain Mapp. 40, 2511-2528. doi: 10.1002/ hbm. 24541

Zilverstand, A., Sorger, B., Slaats-Willemse, D., Kan, C. C., Goebel, R., and Buitelaar, J. K. (2017). fMRI Neurofeedback training for increasing anterior cingulate cortex activation in adult attention deficit hyperactivity disorder. An exploratory randomized, single-blinded study. PLoS One 12:e0170795. doi: 10.1371/journal.pone.0170795

Zuo, X. N., Anderson, J. S., Bellec, P., Birn, R. M., Biswal, B. B., Blautzik, J., et al. (2014). An open science resource for establishing reliability and reproducibility in functional connectomics. Sci. Data 1:140049. doi: 10.1038/ sdata.2014.49

Conflict of Interest: The authors declare that the research was conducted in the absence of any commercial or financial relationships that could be construed as a potential conflict of interest.

Copyright (C) 2019 Seghier, Fahim and Habak. This is an open-access article distributed under the terms of the Creative Commons Attribution License (CC $B Y)$. The use, distribution or reproduction in other forums is permitted, provided the original author(s) and the copyright owner(s) are credited and that the original publication in this journal is cited, in accordance with accepted academic practice. No use, distribution or reproduction is permitted which does not comply with these terms. 